\title{
Sequential Retraction Segregates SGN Processes during Target Selection in the Cochlea
}

\author{
Noah R. Druckenbrod and Lisa V. Goodrich \\ Department of Neurobiology, Harvard Medical School, Boston, Massachusetts 02115
}

\begin{abstract}
A hallmark of the nervous system is the presence of precise patterns of connections between different types of neurons. Many mechanisms can be used to establish specificity, including homophilic adhesion and synaptic refinement, but the range of strategies used across the nervous system remains unclear. To broaden the understanding of how neurons find their targets, we studied the developing murine cochlea, where two classes of spiral ganglion neurons (SGNs), type I and type II, navigate together to the sensory epithelium and then diverge to contact inner hair cells (IHCs) or outer hair cells (OHCs), respectively. Neurons with type I and type II morphologies are apparent before birth, suggesting that target selection might be accomplished by excluding type I processes from the $\mathrm{OHC}$ region. However, because type I processes appear to overshoot into type II territory postnatally, specificity may also depend on elimination of inappropriate synapses. To resolve these differences, we analyzed the morphology and dynamic behaviors of individual fibers and their branches as they interact with potential partners. We found that SGN processes continue to be segregated anatomically in the postnatal cochlea. Although type I-like fibers branched locally, few branches contacted OHCs, arguing against synaptic elimination. Instead, time-lapse imaging studies suggest a prominent role for retraction, first positioning processes to the appropriate region and then corralling branches during a subsequent period of exuberant growth and refinement. Thus, sequential stages of retraction can help to achieve target specificity, adding to the list of mechanisms available for sculpting neural circuits.
\end{abstract}

Key words: auditory; cochlea; cochlear development; refinement; spiral ganglion neuron; target selection

\section{Significance Statement}

During development, different types of neurons must form connections with specific synaptic targets, thereby creating the precise wiring diagram necessary for adult function. Although studies have revealed multiple mechanisms for target selection, we still know little about how different strategies are used to produce each circuit's unique pattern of connectivity. Here we combined neurite-tracing and time-lapse imaging to define the events that lead to the simple binary wiring specificity of the cochlea. A better understanding of how the cochlea is innervated will broaden our knowledge of target selection across the nervous system, offer new insights into the developmental origins of deafness, and guide efforts to restore connectivity in the damaged cochlea.

\section{Introduction}

Animals use an array of circuits to detect the wide variety of cues in their environment. Although all sensory circuits share a ge-

Received June 10, 2015; revised Nov. 2, 2015; accepted Nov. 8, 2015.

Author contributions:N.R.D. and L.V.G. designed research;N.R.D. performed research;N.R.D. and L.V.G. analyzed data; N.R.D. and L.V.G. wrote the paper.

This work was supported by the NIDCD (R01 DC009223 to L.V.G.), the NINDS (T32 NS007484 to N.R.D.), a John and Virginia Kaneb Fellows Award (LVG), and a Mahoney Fellowship (N.R.D.). We thank Dr Thomas Coate (Georgetown University) and Dr Matthew Kelley (NIDCD) for their advice and ideas throughout the project, as well as all members of the Goodrich laboratory for their input along the way and comments on the paper, especially Dr Brikha Shrestha and Andrea Yung; Dr David Corey (Harvard Medical School), who generously shared access to his confocal microscope; Dr Melanie Stefan (Harvard Medical School) for her assistance in selecting the appropriate statistical tests for analysis; and Dr Lai Ding and others at the Harvard Neurodiscovery Center for assistance and access to software.

The authors declare no competing financial interests.

Correspondence should be addressed to Dr. Lisa Goodrich, Harvard Medical School, 220 Longwood Avenue, Boston, MA 02115. E-mail: Lisa_Goodrich@hms.harvard.edu.

DOI:10.1523/JNEUROSCI.2236-15.2015

Copyright $\odot 2015$ the authors $\quad 0270-6474 / 15 / 3516221-15 \$ 15.00 / 0$ neric organization, with information flowing from receptor cells toward ganglion neurons, each circuit's pattern of connectivity is further optimized for the unique demands of the sense. For instance, in the retina, subtypes of amacrine cells and retinal ganglion cells form a large number of cell-type-specific connections within discrete sublaminae, thereby enabling parallel processing of different features of the visual stimulus (Sanes and Zipursky, 2010). In contrast, in the cochlea, most spiral ganglion neurons (SGNs) extend a single unbranched peripheral process toward an inner hair cell (IHC), an organization that is ideal for the rapid and temporally precise transmission of information necessary for sound localization (Meyer and Moser, 2010). Thus, presynaptic and postsynaptic partners must be matched in different ways to create the specialized arrangements found across sensory systems.

Efforts to understand how specificity is established have revealed many possible mechanisms (Sanes and Yamagata, 2009). For example, in the fly eye, subtypes of neurons position their 


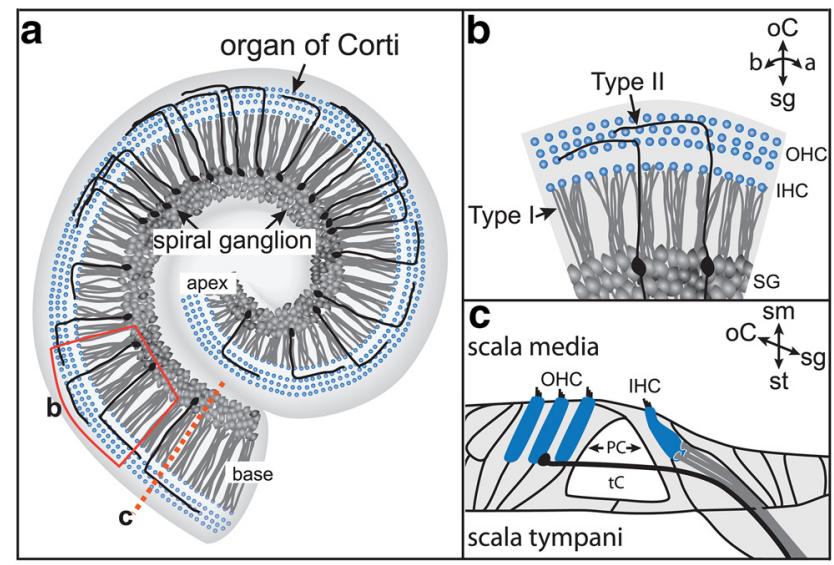

Figure 1. The organization of $S \mathrm{GN}$ innervation in the mature cochlea. $\boldsymbol{a}$, In a flat-mount view of the cochlea, the spiral ganglion and organ of Corti spiral together from the apex to the base. $\boldsymbol{b}$, An enlarged region taken from the red box in $\boldsymbol{a}$. SGN peripheral processes terminate at hair cell targets (blue) in the organ of Corti. Type I SGNs (gray) extend radial fibers that contact a single IHC (gray). Type II SGN (black) peripheral processes instead spiral toward the base, contacting multiple $\mathrm{OHCs}$ (black). $\boldsymbol{c}, \mathrm{A}$ cross-section through the cochlea (a, dashed line) illustrates that type I and II SGN processes are also organized along the scala media-scala tympani axis. a, apex; b, base; $0 \mathrm{C}$, organ of Corti; $s g$, spiral ganglion.

axons in a common region and are then directed toward distinct targets, with different combinations of molecules acting at each step (Pecot et al., 2013). Similarly, in the fly olfactory system, diffusible cues direct axons and dendrites within the antennal lobe, whereas cell-type-specific adhesion molecules help pair presynaptic and postsynaptic partners locally (Hong and Luo, 2014). Classic guidance systems and cell-cell interactions also influence the assembly of visual and olfactory circuits in vertebrates (D'Orazi et al., 2014; Takeuchi and Sakano, 2014). Yet despite recent advances, we still know little about how various strategies are tailored to establish the diversity of wiring that typifies the vertebrate nervous system.

With a stereotyped and simple pattern of connectivity, the cochlea offers an opportunity to learn how targeting occurs across sensory circuits. The sense of hearing depends on the activity of two basic types of SGNs: the type I SGNs, which make radial contacts with a single IHC, and the type II SGNs, which grow past the IHCs and spiral among the outer hair cells (OHCs), forming multiple en passant synapses (Berglund and Ryugo, 1987; Fig. 1). SGNs develop intermingled in the ganglion and extend processes together into the surrounding mesenchyme, eventually penetrating the basilar membrane to reach the auditory sensory epithelium, the organ of Corti. Subsequently, type I and type II processes diverge; however, it is not known how or when each population finds its final targets. Because SGNs with type I or type II morphologies can be recognized embryonically in the mouse, one possibility is that each population is guided directly to the appropriate location (Koundakjian et al., 2007). However, this early differential outgrowth may not be sufficient, because type I SGNs overshoot into the OHC region postnatally (Perkins and Morest, 1975; Echteler, 1992; Simmons, 1994; Huang et al., 2007). Because the exuberant growth coincides with a transient increase in the number of synaptic puncta in the OHCs (Sobkowicz et al., 1986; Huang et al., 2012), target specificity may be achieved instead by elimination of off-target synapses. Additionally, there is a wave of cell death that culls the type II SGN population during this same time frame (Barclay et al., 2011). It is unclear how each of these mechanisms contributes to the final innervation pattern of the cochlea.
To clarify the cellular events that ensure cochlear wiring specificity, we investigated the behavior of individual SGN peripheral processes as they approach their targets in situ. We find that SGN processes are segregated even as they branch locally within the organ of Corti and show behaviors that are inconsistent with a major role for synaptic elimination. Instead, time-lapse imaging studies suggest that targeting involves sequential stages of exploration and retraction, with early cues positioning SGN fibers in the organ of Corti and later cues confining branches to the appropriate region.

\section{Materials and Methods}

Mice. SGNs were labeled by crossing Bhlhb5-Cre (Ross et al., 2010) or Neurog1-CreER ${ }^{T 2}$ mice (Koundakjian et al., 2007) to the AI14 Credependent tdTomato reporter strain (Madisen et al., 2010; no. 007908 The Jackson Laboratory). For time-lapse imaging experiments, male mice with both alleles (ie, Neurog1-CreER ${ }^{T 2}$;AI14) were bred with adult CD1 females (Charles River Laboratories). Noon on the day of the plug was considered embryonic day (E)0. For postnatal collections, the day of birth was called P0. Embryos and pups of either sex were used. Animals were maintained and handled according to protocols approved by the IACUC at Harvard Medical School.

Immunohistochemistry. The following primary antibodies and dilutions were used: rabbit anti-DsRed (Clontech; 1:1000), rabbit antiMyoVI (Proteus; 1:500), and mouse anti-MyoVIIa (Developmental Studies Hybridoma Bank, clone 138.1; 1:200). AlexaFluor-conjugated secondary antibodies raised in donkey were all used at the same concentration (Jackson ImmunoResearch; 1:500). Permeabilization, blocking and antibody hybridization steps were done in phosphate buffer saline (PBS) containing $0.3 \%$ Triton, 3\% bovine serum albumin, and $0.01 \%$ sodium azide. All tissues were dissected, washed in PBS, fixed with $4 \%$ paraformaldehyde in PBS overnight at $4^{\circ} \mathrm{C}$, and then washed in PBS. Dissected cochleae were permeabilized and blocked at room temperature for $2 \mathrm{~h}$. Primary antibody hybridization was performed in the same blocking buffer solution overnight at $4^{\circ} \mathrm{C}$. After washing in PBS (room temperature), secondary antibody incubation was performed in blocking buffer overnight at $4^{\circ} \mathrm{C}$, followed by additional washes the next day in PBS at room temperature. Embryonic cochleae were directly imaged. Postnatal cochleae were transferred through a graded series into $100 \%$ methanol and then placed flush on a glass slide within a rectangular silicon grease reservoir. Methanol within the reservoir was gently aspirated and replaced with a 1:1 solution of methanol-BABB (benzyl alcohol-benzyl benzoate, 1:2 ratio). After partial clearing, this solution was replaced with $100 \% \mathrm{BABB}$, and the silicon grease reservoir was sealed with a glass coverslip.

$3 D$ reconstruction and analysis. To study postnatal refinement, antiDsRed/MyoVIIa double-stained cochleae from animals aged P0 $(n=5)$, P3 $(n=5)$, and P6 $(n=3)$ were collected from at least two separate litters. Confocal $z$-stacks through optically cleared postnatal cochleae were obtained on an Olympus FluoView microscope and then rendered and reconstructed using IMARIS software (Bitplane AG). Processes selected for reconstruction were unambiguously separated from neighbors and could be traced continuously from the proximal mesenchyme to their peripheral terminations in the organ of Corti. Processes and their branches were reconstructed semimanually by guiding both manual and auto-trace outline tools at each plane. Occasionally, individual image planes were manually cropped to retain only the area of interest. The reconstructed $z$-stacks were then rendered to create segmented isosurfaces for each projection with shadows and color to enhance visualization of morphology. The position of each terminal along the scala mediascala tympani (sm-st) axis corresponded to the $z$-position in the confocal stack metadata. Due to small variations between cochlea size and metadata assignments, the $z$-positions of the terminals within each cochlea were assigned a normalized value $\mathrm{Z}$ (norm), where $x$ is the raw $z$-value, $\mu$ is the mean raw $z$-value for all the terminals in a cochlea, and $\sigma$ is the SD:

$$
Z(\text { norm })=\frac{x-\mu}{\sigma} .
$$



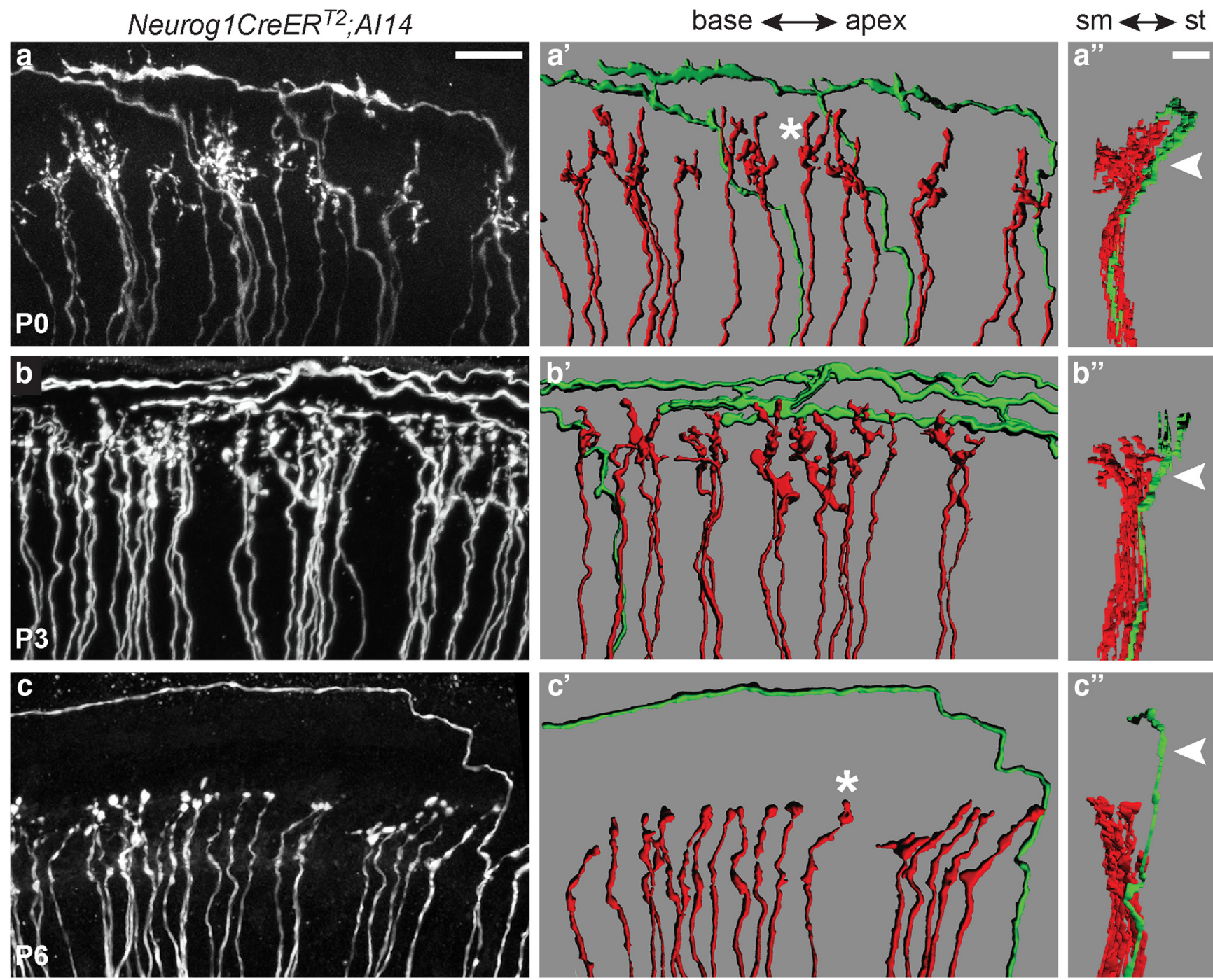

Figure 2. SGN peripheral processes are segregated within the organ of Corti by birth. Flat-mount views of confocal stack projections from anti-DsRed immunostained Neurog1-CreER ${ }^{T 2}$;Al14 cochleae at $\mathrm{PO}(\boldsymbol{a}), \mathrm{P3}(\boldsymbol{b})$, and $\mathrm{P} 6(\boldsymbol{c})$. SGN peripheral processes appear intermingled within the IHC and $0 \mathrm{HC}$ regions at $\mathrm{PO}$ and $\mathrm{P3}$, but obvious type I and type II SGN processes are confined to the IHC or $\mathrm{OHC}$ region, respectively, by $\mathrm{P} 6 . \boldsymbol{a}^{\prime}-\boldsymbol{c}^{\prime}, 3 \mathrm{D}$ reconstructions of the confocal stacks reveal the spiral and radial projections are in fact segregated at $\mathrm{P} 0$ and $\mathrm{P} 3\left(\boldsymbol{a}^{\prime}, \boldsymbol{b}^{\prime}\right)$, similar to what is seen at $\mathrm{P} 6$ $\left(\boldsymbol{c}^{\prime}\right)$. At each stage, individual processes display either radial (red) or spiral (green) morphologies. Additionally, the radial terminals start out highly branched ( $\boldsymbol{a}^{\prime}$, asterisk) but have simpler morphologies by P6 ( $\boldsymbol{c}^{\prime}$, asterisk). $\boldsymbol{a}^{\prime \prime}-\boldsymbol{c}^{\prime \prime}$, Side views of the reconstructions reveal additional organization along the sm-st axis. At birth, the mature organization between radial and spiral projections along this axis has already been established (Movie 1). Scale bars: $\boldsymbol{a}-\boldsymbol{c}, \boldsymbol{a}^{\prime}-\boldsymbol{c}^{\prime}, 20 \mu \mathrm{m} ; \boldsymbol{a}^{\prime \prime}-\boldsymbol{c}^{\prime \prime}, 10 \mu \mathrm{m}$.

Terminals were further classified by whether they arose from radial or spiral processes, as well as their position relative to MyoVIIa-positive hair cells. IHC and $\mathrm{OHC}(\mathrm{r})$ terminals were defined as those that arose from radial fibers and terminated within the volume of MyoVIIa-positive IHCs or OHCs. OHC(s) contacts were defined as the point where spiraling fibers first contact OHCs. Apical (AP) terminals were those ending apical, but not localized to, the MyoVIIa-positive IHC space. Pillar cell (PC) processes terminated between IHC and OHCs without touching either obviously. The branching of each parent process was determined by manually tracing distal terminals in each region back toward the cell body in the ganglion. Only unambiguously singular processes were analyzed.

Organotypic culture. Under sterile conditions, $35 \mathrm{~mm}$ dishes with a 14 $\mathrm{mm}$ glass bottom micro-well (MatTek) were siliconized with Sigmacote (Sigma-Aldrich) for $1 \mathrm{~h}$ at room temperature, air-dried under the hood for 30-60 min, washed with distilled water, and then air-dried once more for $30 \mathrm{~min}$. Next, two small drops of activated Kwik-Sil (World Precision Instruments) were placed $\sim 5 \mathrm{~mm}$ apart on the dish and quickly shaped with a pipette tip before polymerization. The dishes were set aside for $30 \mathrm{~min}$ to allow complete polymerization of silicon elasto- mer. Small squares of electrostatically charged cellulose membrane were evenly affixed to the surface surrounding the glass well using silicone grease and blunted forceps. The dishes were then rinsed with room temperature calcium-free Hank's buffered saline solution supplemented with 20 mM HEPES (HHBS). Finally, two cut pieces of $25-\mu \mathrm{m}$-diameter tungsten wire (Electron Tube Store) were placed within each elastomer droplet at crossing angles. Cochleae from embryos collected from timed pregnant females were dissected in chilled HHBS and placed in the glass bottom dishes, with the base down and the apex skewered with the two crossing pins. Small pieces of devascularized amniotic membrane were draped over the pinned cochleae. The ends of the membrane were pulled taut and then affixed to the charged membrane on either side, thereby ensuring that the cochlea remained in place close to the glass throughout imaging. The dish was then flooded with room temperature organ culture media [defined DF12, Glutamax (Invitrogen), N2 supplement, 25 mM Glucose, 20 mm HEPES, 25 mm sodium bicarbonate, and 5\% FBS (Invitrogen)] to surface tension, and a customized lid with a syringe port was placed on top and sealed with silicone grease. A thin wire thermocouple was threaded through the lid port and placed near the tissue. The imaging chamber was then placed within a heated chamber insert (Har- 


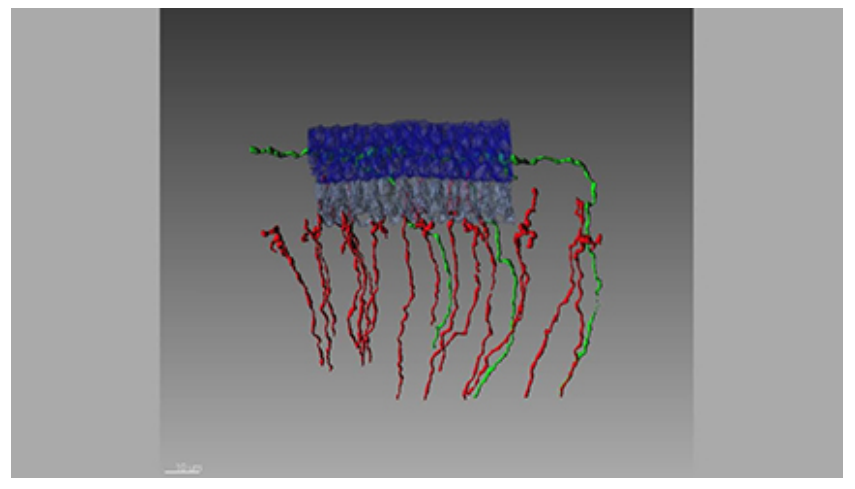

Movie 1. Spiral and radial fibers are segregated in the $P 0$ cochlea. A $3 D$ reconstruction of sparsely labeled SGN fibers and terminals from a Neurog1-CreER ${ }^{T 2}$; Al14 P0 cochlea that was double-stained for DsRed and MyoVIlla. Spiral and radial fibers are color-coded green and red, respectively. Reconstructed MyoVIla-positive IHCs and OHCs are color-coded gray and blue. Toward the end of the movie, the same fibers are depth-coded. Scale bar, $10 \mu \mathrm{m}$.

vard Apparatus) and connected to a custom heated stage controller set to $36.5^{\circ} \mathrm{C}$. The tissue was monitored for $\sim 1 \mathrm{~h}$ during temperature equilibration and then imaged.

Time-lapse imaging and tracking. All time-lapse imaging and tracking studies were done using cochleae harvested from Neurog1-CreER ${ }^{T 2} ; A I 14$ embryos, which enabled visualization of sparsely labeled SGNs and their processes without further immunostaining. Using FluoView software, images were acquired every $10 \mathrm{~min}$ on an upright Olympus confocal microscope with a $60 \times$ oil lens through the entire focal depth of SGN processes $(50-100 \mu \mathrm{m})$ with $z$-steps $\leq 5 \mu \mathrm{m}$. ImageJ was used to transform each time-lapse experiment into a sequence of depth-coded images overlaid with DIC frames. The depth-coded stacks underwent manual branch tracking with MetaMorph software. Processes were selected for analysis if they were $>5 \mu \mathrm{m}$ in length, had an unconnected terminal that was clearly delineable from its neighbors along the $x-y-z$ axes, and could be followed for $\geq 5$ continuous time points. As the most terminal endpoint of each process was tracked, its position with respect to concurrent DIC frames was used to assign its position relative to IHC and OHC regions. Tracking data were exported from MetaMorph as Excel charts containing $x-y$ coordinates, speed, angle, and distance.

Time-lapse statistics and analysis. The angle and distance of all movements between time frames were used to compute mean vector values for each group (ie, E16-E18 vs IHC-OHC) and plotted using Oriana software (Kovach Computing). From these vector pairs, the mean vector angle and length were weighted with the distance traveled. Each group contained $>250$ vector pairs ("instantaneous movements") from three separate imaging experiments. R was used with the "Circstats" package to run circular significance tests. Rao's spacing and Watston's $U^{2}$ tests were used to measure the concentration of values around the mean vector. Differences in mean vector distribution between the study conditions were assessed using Watson's $U^{2}$ multisample test (Li and HoffmanKim, 2008). The directionality of each track was calculated where $d_{t}$ denotes the straight-line distance between the start point and the current position, and $D_{\mathrm{t}}$ is the actual track length from the start length to the current position (Gorelik and Gautreau, 2014).

$$
\text { Directionality }=\frac{\mathrm{d}_{\mathrm{t}}}{D_{\mathrm{t}}} .
$$

The ratios for all tracks in the group were then averaged. The average speed within each subgroup was calculated by taking the grand average of the mean instantaneous speed per track.

Consecutive retraction within each region was defined as any track exhibiting negative angular trajectory (ie, back toward the ganglion or "modiolar") for $\geq 4$ ( $10 \mathrm{~min}$ interval) time points. To measure retraction after entering the $\mathrm{OHC}$ region, processes that began in the IHC region, entered the OHC-region, and retracted within $\leq 4$ time points were classified as retractive. To measure retraction bias along the scala media- scala tympani axis, the midpoint of the $z$-value encompassing the total axon volume was determined at each frame in the movie. Images in the half closer to scala media (red) or scala tympani (green) were pseudocolored and reconstructed into color-coded stacks. These were then manually analyzed for red and green branch retraction in the OHC region. Retraction was again defined as any branch that did not persist in the OHC-region for $>4$ time points. Multiple growth vignettes were compiled from four cochleae from each age. Each vignette lasted for $\geq 3 \mathrm{~h}$.

\section{Results}

\section{SGN fibers are anatomically segregated by birth}

During target selection in the cochlea, type I and type II SGNs extend processes together toward the organ of Corti, and then diverge, with type I SGNs contacting only IHCs and type II SGNs contacting only OHCs. Although neurons with typical type I and type II morphologies can be recognized in fixed tissue as early as E16 (Bruce et al., 1997; Koundakjian et al., 2007), it is unclear whether these early differences also ensure formation of celltype-specific contacts. In fact, type I SGN peripheral processes overshoot extensively within the organ of Corti during the first postnatal week (Perkins and Morest, 1975; Echteler, 1992; Simmons, 1994; Huang et al., 2007), possibly making transient synaptic contacts with OHCs that are absent by P6 (Huang et al., 2012). This is consistent with the possibility that specificity is determined by synaptic elimination, such that only type I-IHC and type II-OHC synapses are retained.

Efforts to understand how SGNs find their targets have been hampered due to the limitations of traditional methods for visualizing cochlear connectivity. Histochemical labeling methods are not amenable to 3D imaging, making it difficult to map terminal position in the organ of Corti, where hair cells and supporting cells are closely packed. Differential uptake of fluorescent dyes, such as tetramethylrhodamine dextran (TMRD) offers one solution. However, although type I, but not type II SGNs, can be TMRD-labeled at P6 (Huang et al., 2007), younger neurons may not be as selective, and there is no independent cell-type-specific marker that can be used to confirm identity at earlier stages, especially because the only known marker for mature type II SGNs, peripherin, is expressed transiently in all SGNs (Hafidi et al., 1993). This is a problem, as TMRD could label other axons passing through the eighth nerve, such as efferents from the hindbrain, sympathetic afferents, or occasional type II SGNs. Additionally, this method does not permit resolution of individual processes, so it has not been possible to determine which cells an individual SGN contacts as it seeks potential synaptic partners.

To gain improved resolution of wiring in the developing cochlea, we combined sparse genetic labeling with optical sectioning and $3 \mathrm{D}$ reconstruction. SGNs were labeled by crossing Neurog1-CreER ${ }^{T 2}$ mice (Koundakjian et al., 2007) to a Credependent tdTomato reporter strain (AI14; Madisen et al., 2010). In Neurog1-CreER ${ }^{T 2}$;AI14 animals, Cre is sporadically active even in the absence of tamoxifen, permitting sparse yet random labeling of SGNs throughout the cochlea (Lu et al., 2014). Because there is no history of Neurogl expression in the hindbrain neurons that extend efferent projections into the cochlea (Yu et al., 2013), this approach allowed us to focus on afferent innervation exclusively. Within the labeled population, peripheral processes were categorized based on classic anatomical criteria (Berglund and Ryugo, 1987; Fig. 1c). Most obviously, type I fibers are radial, whereas type II fibers spiral toward the base of the cochlea. In addition, the fibers are segregated within the depth of the organ of Corti, which sits on the floor of the cochlear duct, with the fluid-filled chambers scala tympani beneath and scala media above. Type I fibers are situated closer to scala media, and 

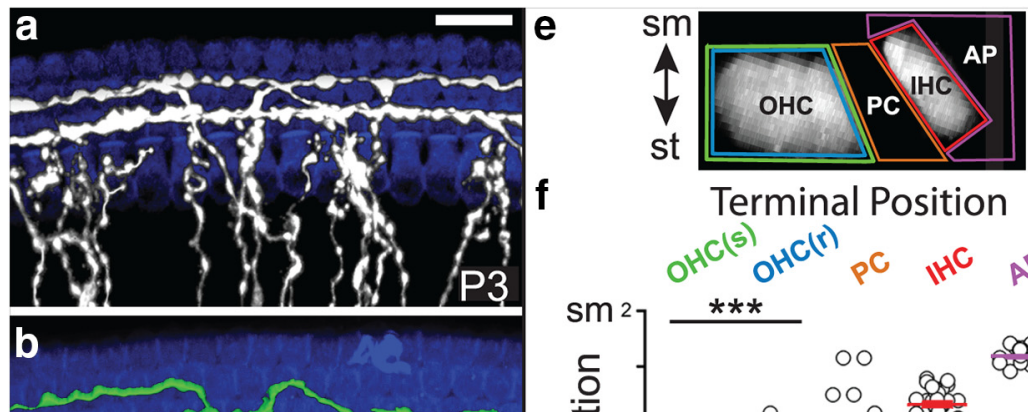

\section{f}
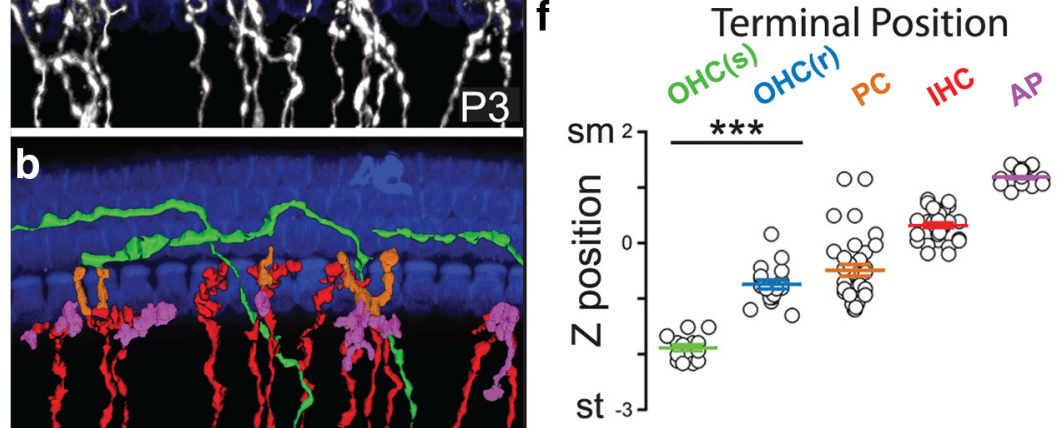

C

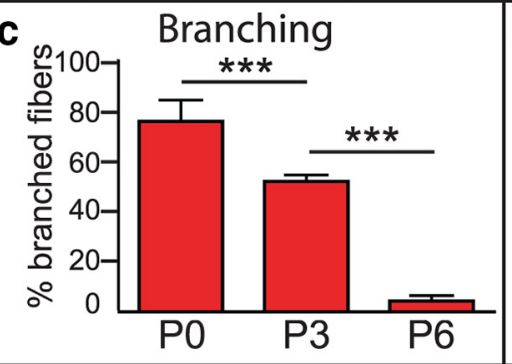

g
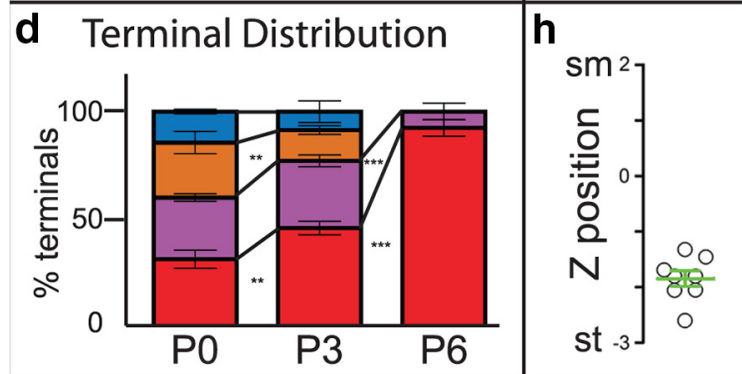

$\emptyset$
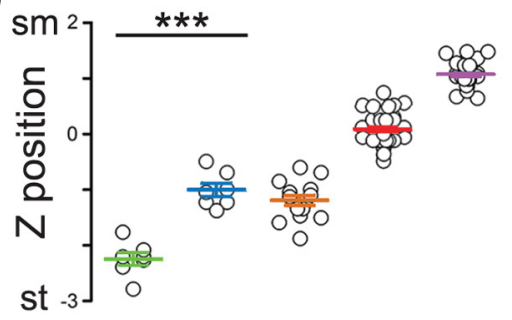

Figure 3. Branches from radial fibers make off-target endings that are progressively lost postnatally. $\boldsymbol{a}, \mathrm{A}$ flat-mount view of a P3 Neurog1-CreER ${ }^{T 2}$;Al14 cochlea with sparsely labeled SGNs (gray) that was immunostained for DsRed to label processes and MyoVlla to label IHCs and $\mathrm{OHCS}$ (blue). $\boldsymbol{b}, 3 \mathrm{D}$ reconstructions of individual radial (red) or spiral (green) fibers were generated, and branches were color-coded according to their origin (radial or spiral) and final position, with branches associated with pillar cells (orange), IHCs (red), or instead ending just apical to the IHCs (purple). In this example, no radial fiber branches contacted an OHC. None of the spiral fibers extended branches outside of the $\mathrm{OHC}$ region at any age (P0: 13 spiral fibers; $\mathrm{P} 3: 7$ spiral fibers; $\mathrm{P} 6: 8$ spiral fibers). c, Quantification of branching of fibers at IHCS over time. Branching decreased significantly between PO and P3, and between $\mathrm{P} 3$ and P6 (P0: 5 cochlea, 54 fibers; P3: 5 cochlea, 65 fibers; $\mathrm{P} 6: 3$ cochlea, 66 fibers; ${ }^{* * *} p<0.0001$, one-way ANOVA with Bonferroni correction). $\boldsymbol{d}$, Quantification of radial terminal distribution over developmental time. At $\mathrm{PO}$, a low percentage of branches contacted IHCs (red), with most branches (88/129) instead making off-target contacts apical to the hair cell (purple), with pillar cells (orange), or with $\mathrm{OHCs}$ in the first row (blue). The percentage of off-target endings decreased at P3 (55/104) and by P6 (4/41), all branches were confined to the IHC region, with just a handful ending in a slightly apical position. Averages of terminal distribution across cochleae are shown (P0: 4 cochleae, 129 terminals; $\mathrm{P3}: 4$ cochleae, 104 terminals; $\mathrm{P} 6: 3$ cochleae, 41 terminals; ${ }^{* *} p<0.005,{ }^{* * *} p<0.0001$, one-way ANOVA with Bonferroni correction). $\boldsymbol{e}$, A side-view of the organ of Corti showing the regions used to classify and count SGN terminals. The position of each ending was mapped and color-coded according to its proximity to an $\mathrm{OHC}$ (green for spiral fibers; blue for radial fibers), $\mathrm{PC}$ (orange), IHC (red), or apical to an IHC (purple). $\boldsymbol{f}-\boldsymbol{h}$, The $z$-position of each terminal was plotted along a normalized sm-st axis and grouped by type. Branches from radial fibers contacted IHCs close to sm, whereas spiral fibers contacted $\mathrm{OHCs}$ close to st. Off-target endings occupied intermediate positions, except for the branches that extended apically near the $\mathrm{IHC}$. Thus, the occasional branches from radial fibers that reached the first row of $\mathrm{OHCS}$ $[\mathrm{OHC}(\mathrm{r})]$ were significantly closer to $\mathrm{sm}$ than the spiral fibers. Because branches were at a similar $x$-y position, these differences are independent of the slight tilt of the organ of Corti. Off-target radial terminals were mostly absent by P6 (P0: 4 cochlea, 144 terminals; $\mathrm{P3}: 4$ cochlea, 107 terminals; P6: 3 cochlea, 49 terminals; ${ }^{* * *} p<0.0001$, one-way ANOVA with Bonferroni correction). Scale bar: $\boldsymbol{a}, 20 \mu \mathrm{m}$. All error bars indicate SEM.

the type II fibers are closer to scala tympani. This system of categorization avoids the possible confounds of transient ectopic expression of cell-type-specific markers in developing neurons, which occurs both in the cochlea (Hafidi et al., 1993; Pan et al.,
PO

2012) and in other regions of the vertebrate nervous system (Azim et al., 2009).

Confocal imaging through the whole mid-base region of cochleae from P0, P3, and P6 animals confirmed a dramatic change in the pattern of overall cochlear wiring over the first postnatal week, with overlapping projections at $\mathrm{P} 0$ gradually becoming segregated to IHC or OHC regions by P6 (Fig. 2a-c). To classify individual peripheral processes, we reconstructed single fibers from confocal stacks and color-coded them according to whether they followed radial (red) or spiral (green) trajectories (Fig. $2 a^{\prime}, b^{\prime}$; Movie 1 ); no other trajectories were observed. Whereas all of those with type II morphologies spiraled exclusively among the OHCs, a subset of radial type I-like processes extended beyond the IHC region at $\mathrm{P} 0$ and $\mathrm{P} 3$, often branching extensively (Fig. $2 a^{\prime}, b^{\prime}$ ). Although top-down views suggested a high degree of overlap, these two populations were actually clearly segregated along the sm-st axis, with radial fibers closer to sm and spiral fibers closer to st (Fig. $2 a^{\prime \prime}-b^{\prime \prime}$ ). This segregation within the depth of the organ of Corti foreshadows the segregation seen at P6, when SGN peripheral processes with definitive type I and type II morphologies were restricted to IHC and $\mathrm{OHC}$ regions, respectively (Fig. $\left.2 c^{\prime}, c^{\prime \prime}\right)$. Thus, despite branching of radial processes beyond the IHCs, the two classes of SGNs appear to be positioned at different depths in the organ of Corti at birth, with type I fibers growing above type II fibers.

\section{Radial peripheral processes branch but make only rare contacts with $\mathrm{OHCs}$} Because type I SGNs appear to be anatomically segregated from type II SGNs even as they overshoot into type II territory, we wondered whether their terminal branches might also display a preference for IHCs from birth. To address this question, we identified individual fibers in anti-DsRed stained Neurog1-CreER ${ }^{T 2}$; AI14 cochleae and mapped where their branches terminated with respect to MyoVIIa-positive hair cells at $\mathrm{P} 0, \mathrm{P} 3$, and P6 (Fig. 3a,b). As expected, fibers with spiral morphologies branched locally, but there were no obvious branches outside of the OHC region at any age $(n=5 \mathrm{P} 0$ cochleae, 5 P3 cochleae, and 3 P6 cochleae; Table 1 shows a summary of all sample sizes and statistical tests used in this study). The radial processes, on the other hand, extended multiple branches toward a variety of cell types, including IHCs (red), $\mathrm{PCs}$ (orange), and occasionally OHCs (not present in this example); some branches also ended apical to the IHC (purple; Fig. 
Table 1. A summary of the sample size, statistical measurements, and tests used to collect and analyze the data shown in specific figures throughout the text

\begin{tabular}{|c|c|c|c|c|}
\hline Measurement & Reference & Groups & Treatment/test & Sample size \\
\hline Percentage of branched processes contacting an IHC over time & Fig. $3 c$ & $\begin{array}{l}\text { P0 vs P3 } \\
\text { P3 vs P6 }\end{array}$ & One-way ANOVA with Bonferroni correction & $\begin{array}{l}\text { P0: } 5 \text { cochleae, } 54 \text { processes } \\
\text { P3: } 5 \text { cochleae, } 65 \text { processes } \\
\text { P6: } 3 \text { cochleae, } 66 \text { processes }\end{array}$ \\
\hline $\begin{array}{l}\text { Distribution of radial terminals within different } \\
\text { subgroups over time }\end{array}$ & Fig. 3d & $\begin{array}{l}P 0 \text { vs } P 3 \\
\text { P3 vs } P 6 \\
\text { Subgroups: IHC, PC, OHC(r), AP }\end{array}$ & One-way ANOVA with Bonferroni correction & $\begin{array}{l}\text { P0: } 4 \text { cochleae, } 129 \text { terminals } \\
\text { P3: } 4 \text { cochleae, } 104 \text { terminals } \\
\text { P6: } 3 \text { cochleae, } 41 \text { terminals }\end{array}$ \\
\hline $\begin{array}{l}\text { Position of terminal subgroups along the sm-st } \\
\text { axis over time }\end{array}$ & Fig. $3 f-h$ & $\mathrm{P} 0$ vs P3 & $\begin{array}{l}\text { Raw data normalized (see Methods)/ } \\
\text { one-way ANOVA with Bonferroni } \\
\text { correction }\end{array}$ & P0: 4 cochleae, 144 terminals \\
\hline & & $\begin{array}{l}\text { P3 vs P6 } \\
\text { Subgroups: IHC, OHC(s), PC, } \\
\quad \text { OHC(r), AP }\end{array}$ & & $\begin{array}{l}\text { P3: } 4 \text { cochleae, } 107 \text { terminals } \\
\text { P6: } 3 \text { cochleae, } 49 \text { terminals }\end{array}$ \\
\hline $\begin{array}{l}\text { Average mean vector "moment" for all tracks over time } \\
\text { within regions (subgroups) }\end{array}$ & Fig. $6 a-d$ & $\begin{array}{l}\text { E16 vs E18 } \\
\text { Subgroups: IHC-, OHC-regions }\end{array}$ & $\begin{array}{l}\text { Weighted angular mean (see Methods)/ } \\
\text { Uniformity tests: Rao's Spacing; Watson's U² }\end{array}$ & $\begin{array}{l}\text { Each age and region: } 3 \text { cochleae } \\
\text { Instantaneous movements (IMs) } \\
\quad \text { per age/region } \\
\text { E16/IHC: } 252 \text { IM } \\
\text { E16/0HC: } 338 \text { IM } \\
\text { E18/IHC: } 329 \text { IM } \\
\text { E18/OHC: } 539 \text { IM }\end{array}$ \\
\hline $\begin{array}{l}\text { Average end-point directionality for all tracks over time } \\
\text { within regions (subgroups) }\end{array}$ & Fig. $6 e$ & $\begin{array}{l}\text { E16 vs E18 } \\
\text { Subgroups: IHC-, OHC-regions }\end{array}$ & Two-way ANOVA with Bonferroni correction & $\begin{array}{l}\text { Each age and region: } 3 \text { cochleae } \\
\text { E16/IHC: } 27 \text { tracks } \\
\text { E16/0HC: } 39 \text { tracks } \\
\text { E18/IHC: } 24 \text { tracks } \\
\text { E18/0HC: } 35 \text { tracks }\end{array}$ \\
\hline $\begin{array}{l}\text { I. Percentage of tracks that show }>4 \text { consecutive retraction } \\
\text { over time within regions (subgroups) }\end{array}$ & Fig. $6 f$ & $\begin{array}{l}\text { E16 vs E18 } \\
\text { Subgroups: IHC-, OHC-regions }\end{array}$ & Two-way ANOVA with Bonferroni correction & $\begin{array}{l}\text { Each age and region: } 3 \text { cochleae } \\
\text { E16IHC: } 23 \text { tracks } \\
\text { E160HC: } 39 \text { tracks } \\
\text { E18 IHC: } 24 \text { tracks } \\
\text { E18 OHC: } 35 \text { tracks }\end{array}$ \\
\hline $\begin{array}{l}\text { II. Percentage of tracks over time within regions (subgroups) } \\
\text { that enter the } \mathrm{OHC} \text { region and then rapidly retract }\end{array}$ & Fig. $6 f$ & $\begin{array}{l}\text { E16 vs E18 } \\
\text { Subgroups: IHC-, OHC-regions }\end{array}$ & $\chi^{2}$ & $\begin{array}{l}\text { E16: } 46 \text { tracks, } 4 \text { cochleae } \\
\text { E18: } 38 \text { tracks, } 3 \text { cochleae }\end{array}$ \\
\hline $\begin{array}{l}\text { The average track speed ( } \mu \mathrm{m} / \mathrm{min} \text { ) over time within } \\
\text { regions (subgroups) }\end{array}$ & Fig. $6 g$ & $\begin{array}{l}\text { IHC: E16 vs E18 } \\
\text { OHC: E16 vs E18 }\end{array}$ & $t$ test & $\begin{array}{l}\text { Each age and region: } 3 \text { cochleae } \\
\text { E16/IHC: } 27 \text { tracks } \\
\text { E16/0HC: } 39 \text { tracks } \\
\text { E18/IHC: } 24 \text { tracks } \\
\text { E18/0HC: } 35 \text { tracks }\end{array}$ \\
\hline $\begin{array}{l}\text { The percentage of tracks that persists within the } \mathrm{E} 18 \mathrm{OHC} \\
\text { region over time analyzed by sm-st position }\end{array}$ & Fig. 8 & $\mathrm{E} 18 / 0 \mathrm{HC}$ sm vs E18/0HC st & $\chi^{2}$ & $\begin{array}{l}\text { E18/0HC sm: } 38 \text { tracks, } 4 \text { cochleae } \\
\text { E18/0HC st: } 20 \text { tracks, } 4 \text { cochleae }\end{array}$ \\
\hline
\end{tabular}

All statistical tests were selected in consultation with a biostatistician.

$3 b, c)$. At P0, $76 \pm 8.49 \%$ of the radial fibers $(n=4$ cochleae $)$ that terminated near an IHC also extended branches toward other cell types. Over time, this proportion gradually decreased, such that by $\mathrm{P} 6$, only $4.33 \pm 2.18 \%$ of the IHC-targeted processes $(n=3$ cochleae) were still branched. Thus at P0, unlike type II SGN processes that are already restricted to their target area, type I-like SGN processes show extensive branching around and beyond the IHCs that is subsequently refined.

By characterizing the distribution of terminals from the radial fibers, we found that branches interact transiently with a variety of cell types, with OHCs comprising only a minor subset (Fig. $3 d$ ). At P0, only $31.9 \pm 4.2 \%$ of the branches $(n=$ 4 cochleae) actually ended near an IHC. The remaining branches consisted of "off-target endings" and were mostly present among the PCs $(25.4 \pm 5.1 \%)$ or close to the IHCs but in an abnormal apical position $(28.4 \pm 1.8 \%)$. In contrast, only $14.3 \pm 1.0 \%$ contacted an $\mathrm{OHC}(\mathrm{OHC}(\mathrm{r}))$, and these contacts were never observed beyond the first row. By P3, an even smaller fraction of branches ended near an OHC (8.5 \pm $5.0 \%)$ or PC $(14.2 \pm 2.0 \% ; n=4$ cochleae $)$, and all branches were confined to the IHC region by P6 ( $n=3$ cochleae), mostly in close proximity to an IHC with just a handful of branches ending slightly apical $(7.5 \pm 3.8 \%)$.
Because type I and type II SGNs are segregated along the sm-st axis in the mature cochlea (Fig. 1c), we wondered whether the distribution of the transient branches extending from the radial fibers was more consistent with a type I or type II identity. We mapped the $z$-position of the spiral fibers as they enter the OHC region, as well as the tips of each branch that could be traced back to a radial fiber. Although in principle the fibers could extend branches anywhere along the depth of the organ of Corti (Fig. 3e), the distribution of branch terminals was remarkably stereotyped at all ages (Fig. $3 f-h$ ): contacts with IHCs were closer to sm, whereas spiraling fibers were closer to st. More strikingly, this was also true for branches from radial fibers that contacted OHCs. The radial fibers also extended branches that did not contact any hair cell, ending instead near PCs or just apical to an IHC. Importantly, although there were fewer contacts between radial fiber branches and non-IHC targets with age (ie, compare Fig. $3 f-h)$, this was not matched by an obvious increase in the number of spiral fibers contacting the OHCs. Together, these data are more consistent with the idea that the small number of contacts between radial fibers and OHCs represent extra branches from type I SGNs rather than nascent type II SGNs that have not yet spiraled. Thus, although radial fibers extend transient branches outside of the IHC region, these branches are at a different depth, 
a
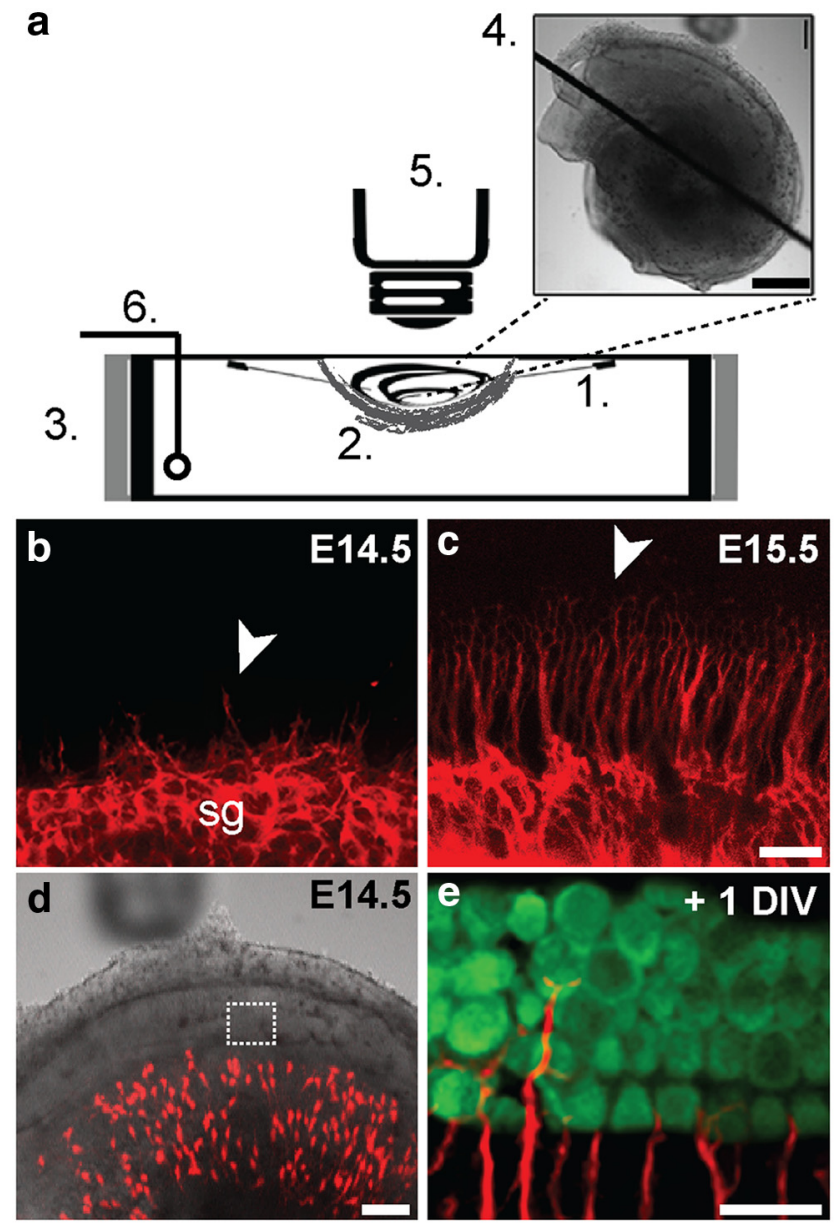

Figure 4. A system for imaging of intact suspended embryonic cochleae. $\boldsymbol{a}, \mathrm{A}$ dissected cochlea is suspended with $25 \mu \mathrm{m}$-diameter-tungsten needles (1), cradled in a net of devascularized amniotic membrane (2), and placed in a modified dish surrounded by resistive heating elements (3). The final preparation (4) was imaged on an upright confocal microscope (5). A thin-wire thermocouple (6) monitors and maintains a physiological temperature throughout the imaging period via feedback to a temperature controller. $\boldsymbol{b}-\boldsymbol{e}$, The cochlea recapitulates normal growth and development in the chamber. In acutely dissected cochleae $(\boldsymbol{b}, \boldsymbol{c})$, SGN processes, which were labeled in bulk with tdTomato (red) by crossing Bh/hb5-cre mice to Al14 reporters, are extending from the spiral ganglion $(\mathrm{sg})$ at E14.5 $(\boldsymbol{b})$ and reach the organ of Corti target region by E15.5 (c). Arrowheads $(\boldsymbol{b}, \boldsymbol{c})$ indicate the front of process outgrowth. This pattern of outgrowth is maintained in the suspended culture preparation (d) of a Neurog1-CreER ${ }^{T 2}$; Al14 cochlea, as evidenced by the presence of sparsely labeled tdTomato-positive SGN processes (e, red) among MyoVI-positive hair cells (e, green) after $1 \mathrm{~d}$ in vitro (+1 DIV; Movie 2). Scale bars: $\boldsymbol{a}$ (inset), $500 \mu \mathrm{m} ; \boldsymbol{b}, \boldsymbol{c}, 20 \mu \mathrm{m} ; \boldsymbol{d}, 100 \mu \mathrm{m} ; \boldsymbol{e}$, $20 \mu \mathrm{m}$.

rarely contact OHCs, and never reach beyond the first row. These findings suggest that radial fibers are unlikely to be the source of the extra synapses present throughout all three rows of OHCs during the first postnatal week (Huang et al., 2012). Instead, the distribution of endings is more consistent with the idea that type I SGNs extend multiple branches beyond the IHC region which are then removed, leaving only contacts with IHCs.

\section{The OHC environment is inhospitable to peripheral process invasion at $\mathrm{E} 16$}

Our studies suggest that target selection involves at least two stages: early differential outgrowth (Koundakjian et al., 2007) followed by local branching and refinement of processes that are already segregated along the sm-st plane (Figs. 2, 3). Each of these steps could occur through a number of ways, includ-

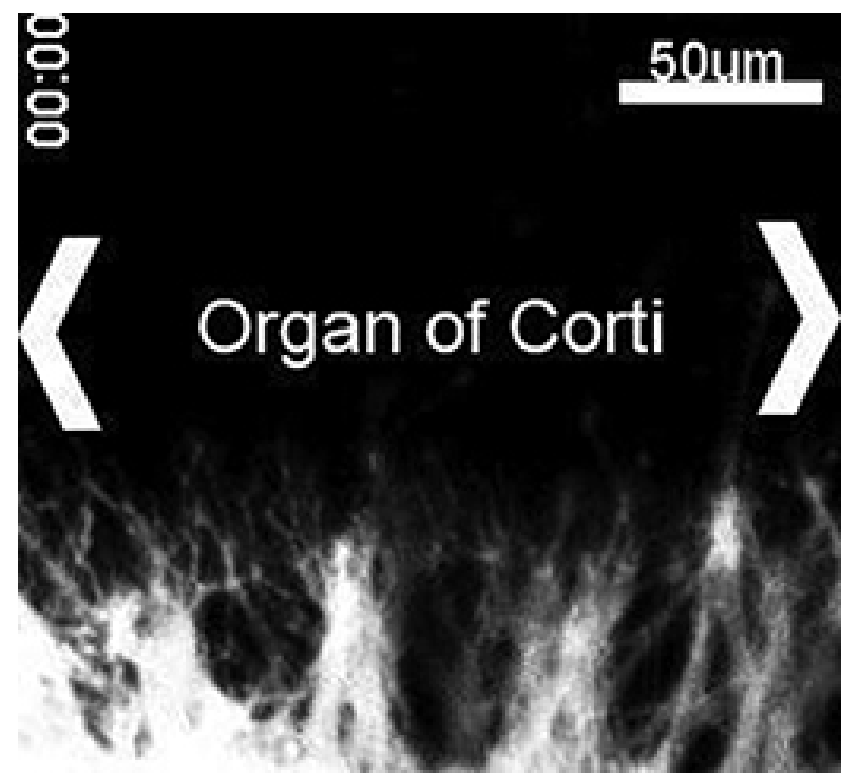

Movie 2. Growth of SGN processes in an E15 cochlear explant. Time-lapse movie of a suspended organotypic culture of an E15 Bh/hb5-cre; $A / 14$ cochlea imaged after 12 h in culture. In contrast to what happens in typical $2 \mathrm{D}$ explants, processes remain within the cochlea and interact with cells in the organ of Corti (white wedges), including their natural synaptic partners. A magnified region from this preparation (rectangle) is RGB coded by depth to illustrate preservation of the dynamic $3 \mathrm{D}$ environment in live tissue. Time, hh:mm. Scale bar, $50 \mu \mathrm{m}$.

ing increased attraction between the "right" partners and repulsion from the "wrong" partners (Sanes and Yamagata, 2009). However, developing axons exhibit local and dynamic changes in behavior, making it difficult to infer cellular mechanism from fixed preparations. Therefore, we used a timelapse imaging system to study how individual SGN processes locate their appropriate partners within their natural context, ie, in growing, suspended, and intact cochleae for $>24 \mathrm{~h}$ (Fig. $4 a)$. Previously, we used this system to examine the initial outgrowth of all SGN processes through the mesenchyme (Appler et al., 2013). To see whether the same system might permit analysis of target selection, we confirmed that the cultured cochleae maintain their normal 3D structure and that the SGN processes grow toward the MyoVI-positive hair cells as they do in vivo (Fig. 4b-e; Movie 2). By using cochleae from Neurog1$\mathrm{CreER}^{T 2}$;AI14 animals and adapting the method for older ages, this system enabled high-resolution imaging of individual SGN processes and their branches as they approach and interact with cells in the organ of Corti.

Because the analysis of fixed tissue confirmed dramatic changes in the branching pattern of SGN processes as they grow to and within the organ of Corti (Fig. 3), we examined the dynamic behaviors of individual branches extending from SGN processes, starting at E16 ( $n=3$ cochleae), when neurons with type I and type II morphologies are first apparent (Koundakjian et al., 2007). At E16, the PCs that separate the developing IHCs from the OHCs can be identified with DIC optics (Fig. 5a). Using the most medial PC as a border, we manually tracked the distal tip of each growing branch that met predefined criteria (see Materials and Methods; Movie 3). Trajectories were inferred by mapping the position of the tip from frame to frame. This analysis revealed a wide variety of trajectories characterized by frequent extension and retraction, as well as changes in direction (Fig. 5b), consistent with the idea that SGN processes are highly exploratory as they navigate. 

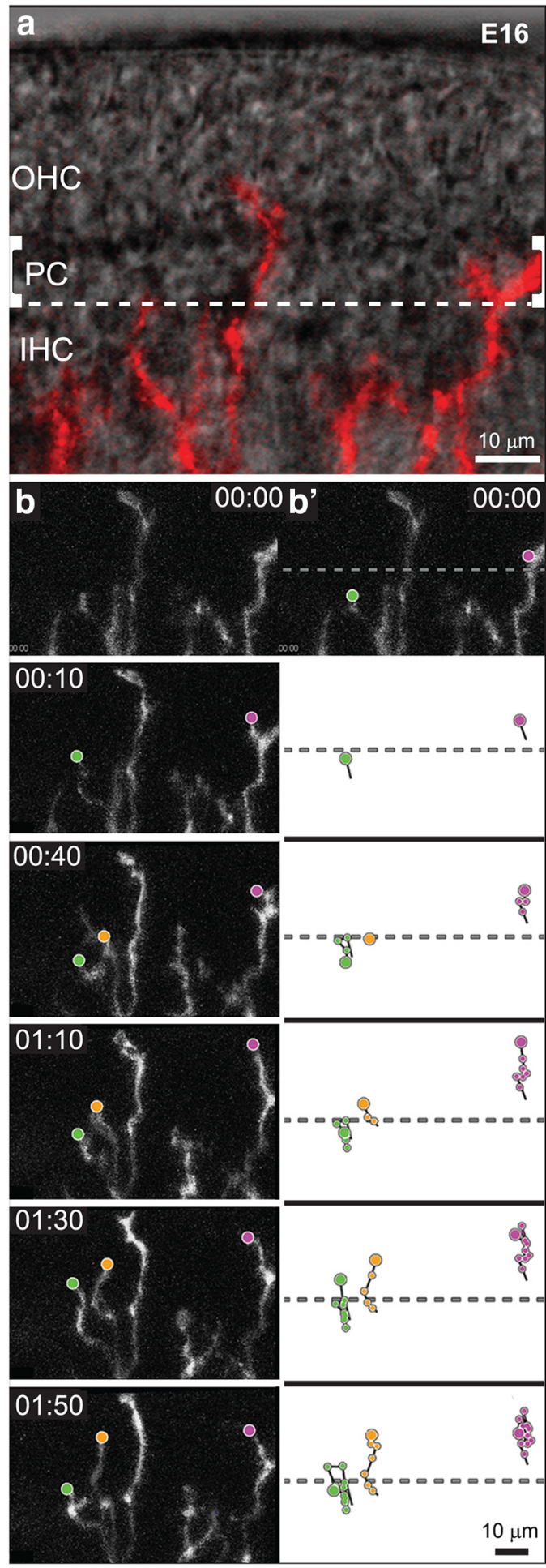

Figure 5. Time-lapse imaging of SGN processes reveals a variety of behaviors within IHC and $\mathrm{OHC}$ regions. $\boldsymbol{a}$, An overlay of bright-field and fluorescent images illustrates the position and morphology of SGN processes (red) in a cultured E16 Neurog1-CreER ${ }^{22}$;Al14 cochlea at the start of imaging. Pillar cells $(\mathrm{PC})$ separate the IHCs from the developing $\mathrm{OHCS}$. SGN peripheral process trajectories were analyzed in the $\mathrm{IHC}$ or $\mathrm{OHC}$ region, using the $\mathrm{PC}$ to delineate the border (dashed lines). $\boldsymbol{b}$, A montage of frames reveals the types of movement exhibited by $\mathrm{SGN}$ processes over several hours of development (Movie 3; time is displayed as hours:minutes). Trajectories were constructed by marking the position of each tip (dots) in successive frames. Processes exhibited a variety of behaviors. For instance, the process illustrated in green crosses the $\mathrm{PC}$ border, but then retracts back to the IHC region; a nearby process advances steadily away from the modiolus (yellow); and a process already in the $\mathrm{OHC}$ region makes only short movements that result in little net movement (magenta). Scale bars, $10 \mu \mathrm{m}$.

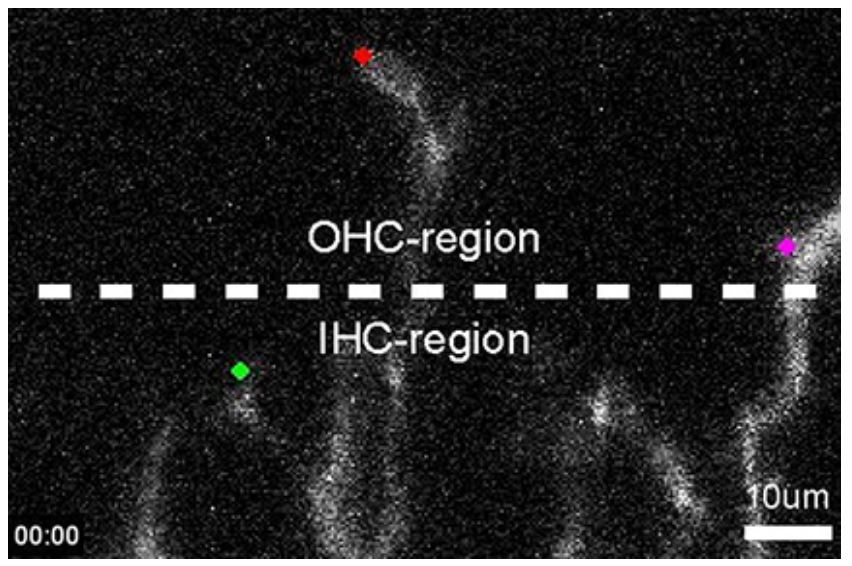

Movie 3. SGN processes exhibit dynamic behaviors in the E16 cochlea. An example of outgrowth behavior in an E16 Neurog1-CreER ${ }^{T 2} ; A / 14$ cochlea that was imaged every $10 \mathrm{~min}$. Processes (gray) were tracked manually along their distal tips (colored dots) and were later assigned to either $\mathrm{IHC}$ or $\mathrm{OHC}$ regions (dashed line), as determined using landmarks in DIC images. Scale bar, $10 \mu \mathrm{m}$.

To learn how the tips of the SGN branches move as they interact with potential targets, all of the tracks in the IHC or OHC region were aligned on a common origin. When viewed this way, it was clear that in the IHC region (Fig. 6a), branches no longer followed a strictly radial pattern of growth away from the modiolus of the ganglion (ie, "amodiolar") but instead began to make frequent withdrawals from the $\mathrm{OHC}$ region (ie, "modiolar"). To quantify this shift in behavior, we measured the direction, velocity, and length of each instantaneous movement and then plotted the average resultant vector, as shown in mean vector plots (Fig. $6 a$ ). This analysis confirmed that the average movement of individual SGN branches was directed toward the modiolus, ie, retraction (Fig. $6 a$, red arrow). However, there was considerable variance within this population, as many movements were also in the opposite direction, ie, extension. Indeed, the overall trajectories were quite directed, as measured by dividing the distance traveled by the actual length of the trajectory (Fig. 6e). Moreover, individual branches often made consecutive retractive movements, with $44 \%$ making at least four consecutive withdrawals, even while in the IHC region ( 10 of 23 tracks, $n=3$ cochleae; Fig. $6 f$ ). These data suggest that most SGN branches either extend toward or retract from the OHCs, with little movement laterally.

Further analysis confirmed that retraction is a prominent feature of growth in the E16 cochlea. Trajectories were grouped according to how far the tip of each branch moved from the start of the movie to the end (ie, distance to origin), as well as the direction of this movement (ie, toward or away from the OHCs; Fig. 7). This analysis revealed three basic types of behavior. Some branches advanced (yellow), similar to the directed outgrowth apparent en route to the organ of Corti. Many others followed obviously retractive paths (green), resulting in a strong net withdrawal from the OHC region. Finally, a subset of branches appeared to explore the region (magenta): though still motile, they made little overall progress, due in part to frequent retractions. This variety of behavior contrasts with the highly directed mode of advancement apparent at earlier stages (Appler et al., 2013), and suggests that individual branches alter their behavior and begin to make many retractive movements as they reach the organ of Corti.

Given the clear increase in retractive movements upon reaching the IHC region, we posited that SGN branches may be posi- 


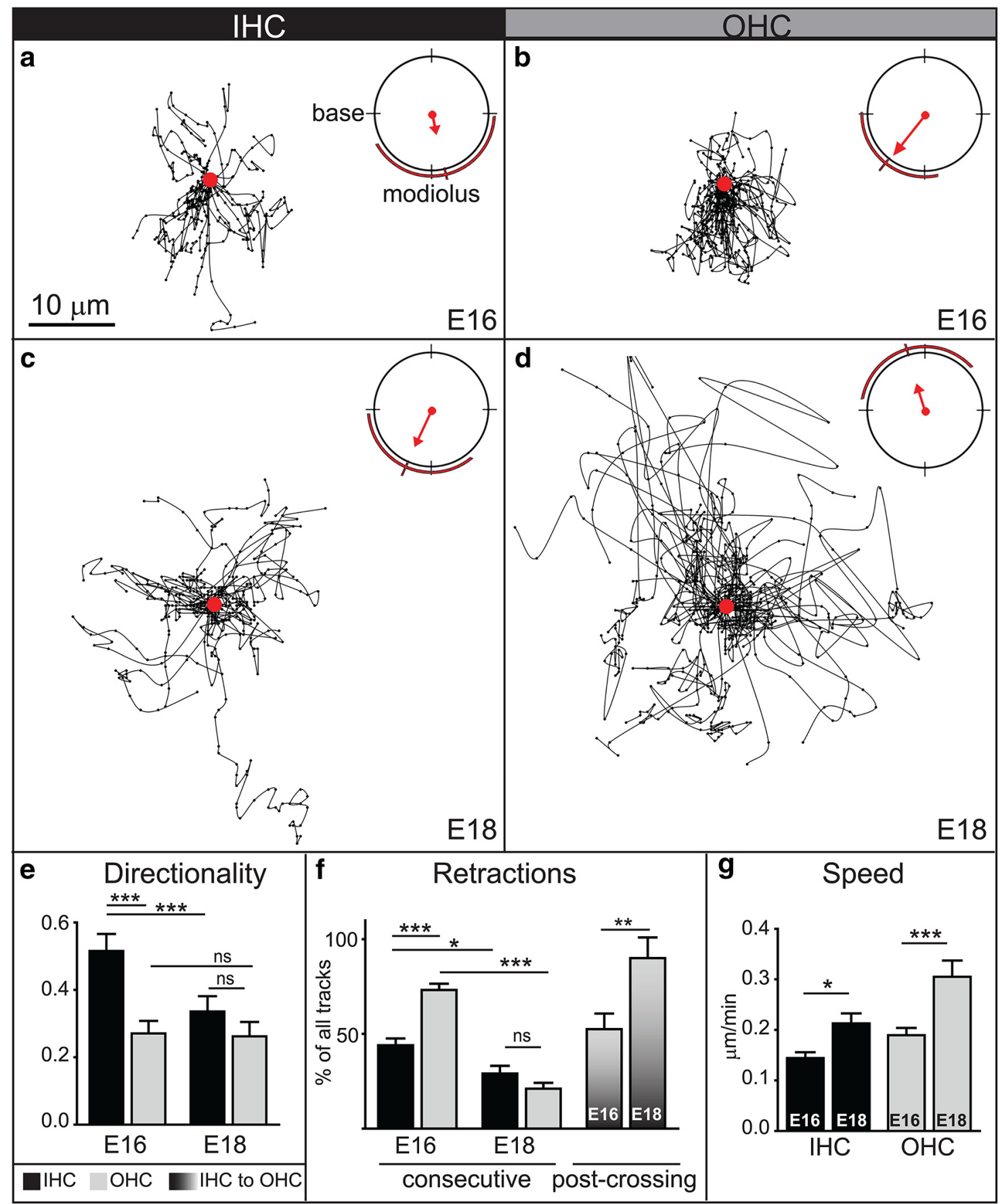

Figure 6. Time-lapse imaging reveals distinct behaviors of SGN peripheral processes as they approach and explore the IHC and $0 \mathrm{HC}$ regions at E16 and E18. $\boldsymbol{a}-\boldsymbol{d}$, Trajectories at E16 $(\boldsymbol{a}, \boldsymbol{b})$ and E18 $(\boldsymbol{c}, \boldsymbol{d})$ within IHC $(\boldsymbol{a}, \boldsymbol{c})$ or OHC regions $(\boldsymbol{b}, \boldsymbol{d})$ were plotted on a common origin (red dot), with the base to the left and the modiolus down. Mean vector plots (upper right hand corners) reflect the average angular mean of all frame-to-frame movements (weighted by speed), as represented by a red arrow. Variance is illustrated by red lines outside the plot. At E16, many tracks point toward the modiolus, particularly in the $\mathrm{OHC}$ region $(\boldsymbol{b})$. AtE18, the greater density of tracks immediately around the origin suggests more local exploration $(\boldsymbol{c}, \boldsymbol{d})$, likely involving many retractive movements since the mean vector of $\mathrm{E} 18 \mathrm{IHC}$ tracks still points toward the modiolus (c). In contrast, the mean vector in the $\mathrm{OHC}$ region is shifted away from the modiolus, with many of the fastest movements directed toward the base (d). (Each age and region: 3 cochleae; number of instantaneous movements: E16/IHC: 252; E16/0HC: 338; E18/HC: 329; E18/0HC: 539. All vector means $p<0.01$ by Rao's spacing test and Watson's $U^{2}$ test. All groups have significantly different vector distributions with $p<0.05$ as determined using Watson's $U^{2}$ multisample test.) $e$, Directionality was quantified by dividing the distance traveled by the actual length of the trajectory and average for all trajectories in the IHC (black bars) or OHC (gray bars) region at E16 and E18. SGN processes growing in the IHC region at E16 were significantly more directed than those in the $\mathrm{OHC}$ region or in either region at E18. (Each age and region: 3 cochleae; number of tracks: $\mathrm{E} 16 / \mathrm{HC}: 27 ; \mathrm{E} 16 / 0 \mathrm{HC}: 39 ; \mathrm{E} 18 / \mathrm{IHC}: 24$; $\mathrm{E} 18 / \mathrm{OHC}: 35 ;{ }^{* * *} p<0.0001$ by one-way ANOVA with Bonferroni correction). $f$, The percentage of tracks that showed consecutive retractive movements was calculated in each region and at each age. A higher percentage of tracks showed long episodes of retraction in the $\mathrm{OHC}$ region than the $\mathrm{IHC}$ region at E16. By E18, the percentage of tracks with consecutive retractions was significantly reduced. (Each age and region: 3 cochleae; number of tracks: E16/IHC: 23; E16/0HC: 39; E18/IHC: 24; E18/0HC: 35; ${ }^{*} p<0.05,{ }^{* * *} p<0.0001$, by one-way ANOVA with Bonferroni correction). However, the $\mathrm{OHC}$ region became even less permissive for entry, as evidenced by the significant increase in the percentage of $\mathrm{SGN}$ peripheral processes that retracted after crossing from the IHC region into the $\mathrm{OHC}$ region (graded bars) at E18 compared with E16. (E16: 4 cochleae, 46 tracks; E18: 3 cochleae, 38 tracks; ${ }^{* *} p<0.005, \chi^{2}$ test). $\boldsymbol{g}$, The average speed of SGN peripheral processes was significantly greater at E18 than at E16 in both regions (same $n$ values as in $e_{;}^{* *} p \leq 0.005, t$ test). Scale bar, $10 \mu \mathrm{m}$. All error bars are SEM. 


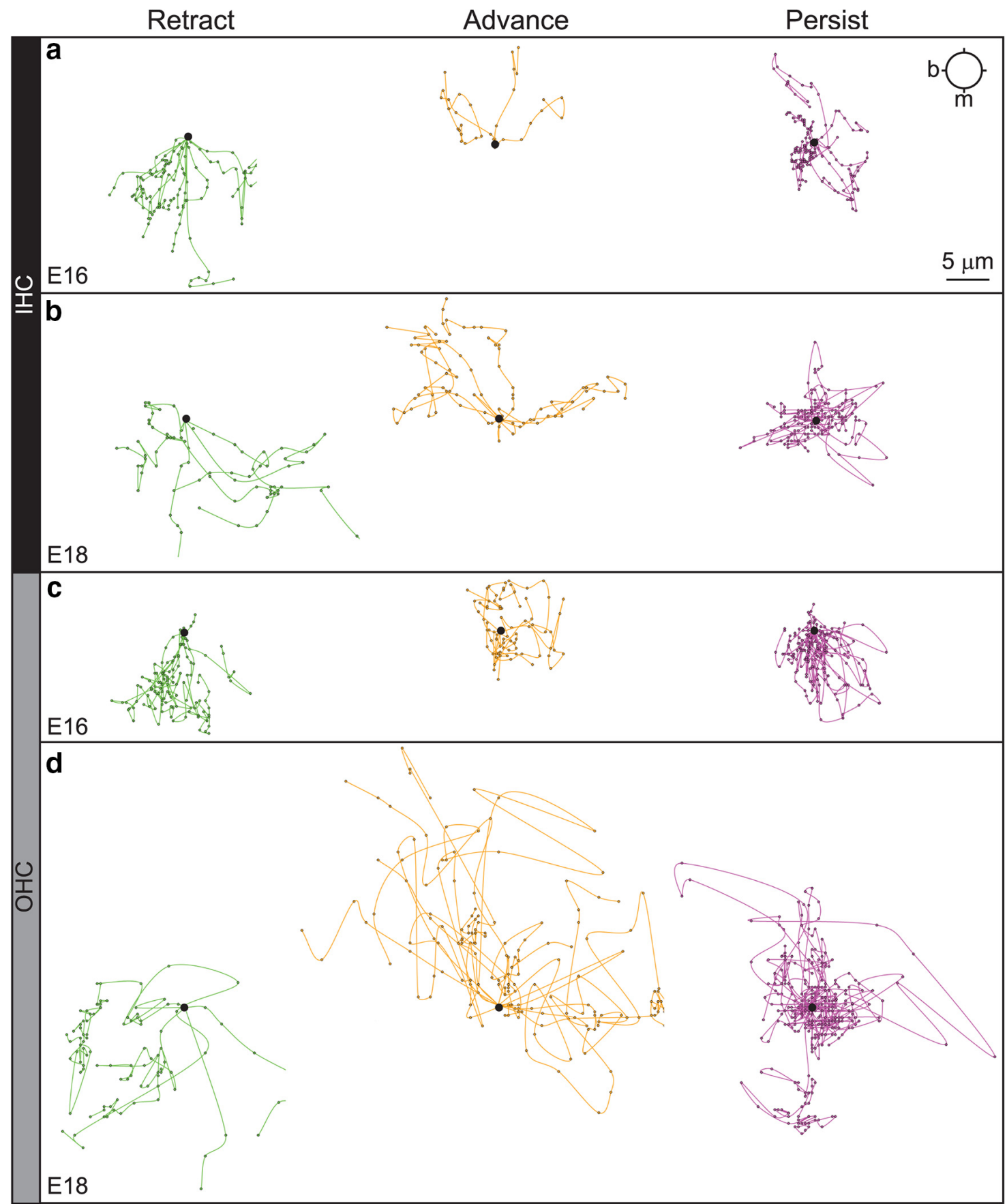

Figure 7. SGN processes follow different paths within each region that change over time. Origin plots for the same tracks shown in Figure 6, further separated into groups based on net distance and end-point. Specifically, the mean distance to origin (DTO) for each group was calculated, and any track below the mean was classified as persistent. Tracks above the mean DTO were classified as retracting if their end-point was modiolar to their start-point. Similarly, advancing tracks had above average DTO and an end-point amodiolar to the start. AtE16 (a, $\boldsymbol{c}$ ), instantaneous withdrawals toward the modiolus were common in those tracks that ultimately retracted in both the $\mathrm{HHC}$-region $(\boldsymbol{a}$, green) and $\mathrm{OHC}$-region ( $\boldsymbol{c}$, green), as well as those that persisted in the $\mathrm{OHC}-$ region ( $\boldsymbol{c}$, magenta), suggesting that even those processes that did not move far from the origin made frequent retractive movements. Advancing processes in the IHC region appeared directed (a, yellow), especially relative to those in the $\mathrm{OHC}$ region ( $\boldsymbol{c}$, yellow). At E18 $(\boldsymbol{b}, \boldsymbol{d})$, movements in the IHC-region $(\boldsymbol{b})$ seemed less directed, with more lateral extensions in the Retract ( $\boldsymbol{b}$, green) and Advance $(\boldsymbol{b}$, yellow) groups and a high density of movements about the origin in the Persist group ( $\boldsymbol{b}$, magenta). In the $\mathrm{OHC}$-region, the occasional Retracting process (d, green) moved very quickly and directly toward the modiolus. Processes in the Advance group ( $\boldsymbol{d}$, yellow) made rapid movements away from the modiolus, often biased toward the base. Processes in the Persist group ( $\boldsymbol{d}$, magenta) circled the origin, with more movements pointed amodiolar and/or toward the base than at E16, when retractive movements clearly dominated ( $c$, magenta). Base (b) is to the left; the modiolus ( $\mathrm{m}$ ) is toward the bottom. Scale bar, $5 \mu \mathrm{m}$.

tioned by a combination of attraction to the organ of Corti and repulsion from the $\mathrm{OHC}$ region. In support of this idea, retractive movements were even more common in the $\mathrm{OHC}$ region, as reflected in a significantly longer mean vector and less variance (Fig. 6b). For example, even those branches that showed little net movement still made many individual retractions in the OHC region (Fig. $7 c$, magenta), and the branches that advanced (Fig. $7 c$, yellow) appeared less directed than those in the IHC region (Fig. 7a, yellow). Altogether, almost three quarters of the tracks (25 of 34 tracks, $n=3$ cochleae) showed more than four consec- 


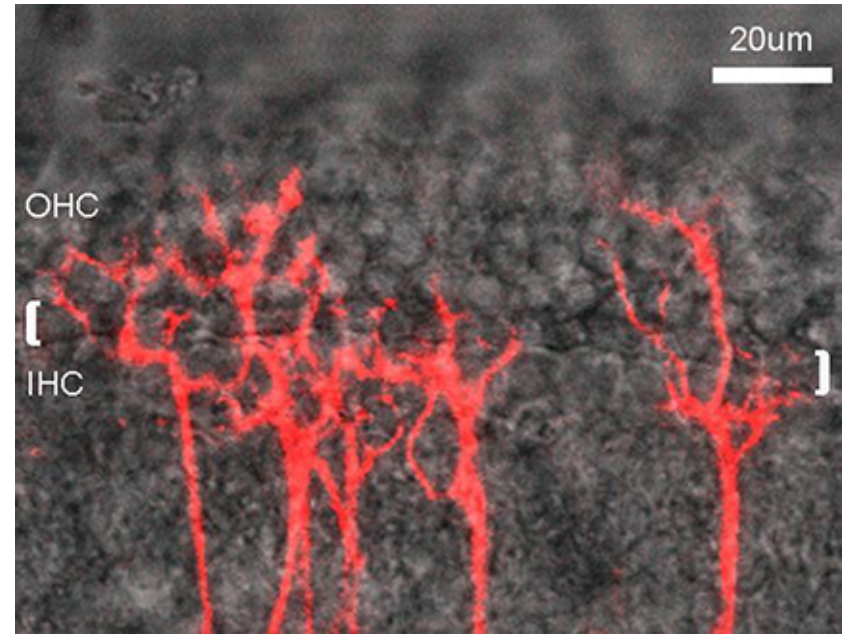

Movie 4. SGN targeting behavior in the E18 cochlea. An example of outgrowth behavior in an E18 Neurog1-CreER ${ }^{T 2}$; Al14 cochlea overlaid with updating bright-field DIC image; images were collected every $10 \mathrm{~min}$. The $\mathrm{OHCs}$ and IHCs are clearly demarcated by the pillar cell region (white brackets). Scale bar, $20 \mu \mathrm{m}$.

utive retractions, which was significantly greater than in the IHC region (Fig. $6 f$ ). The average speed was also significantly increased (Fig. $6 g$ ). Thus, within the OHC region, more SGN branches retract steadily, and each retractive movement is faster than in the IHC region. Importantly, retraction was particularly common for the subset of branches that entered the $\mathrm{OHC}$ region from the IHC region: $52 \pm 4.1 \%$ of these branches $(n=4$ cochleae; 46 branches were scored overall) began to retract within 40 min of crossing the PC border (Fig. 6f). Thus, individual branches exhibit a mixture of forward and reverse growth upon reaching the organ of Corti, as if retraction is induced in response to a repulsive cue emanating from the $\mathrm{OHC}$ region.

\section{Processes segregated to the IHC or $\mathrm{OHC}$ region show distinct responses during local target selection}

Our results suggest that SGN peripheral processes are positioned in the IHC region because the $\mathrm{OHC}$ region is not permissive to outgrowth: branches begin to retract as they approach the $\mathrm{OHC}$ region and leave this region quickly after they enter. However, this early segregation is not sufficient, as ultimately, type I SGNs must find their IHC targets and the type II SGNs must grow among the OHCs. To determine the cellular mechanisms responsible for this later stage of target selection, we performed a series of imaging experiments at E18, when processes are largely segregated and are entering a phase of branching and refinement.

SGN behaviors were significantly changed at E18, with branches in the IHC region exploring locally and those in the $\mathrm{OHC}$ region instead advancing rapidly. For instance, within the IHC region, the branches became more exploratory than they were at E16, as evidenced by a high density of movements surrounding the origin and more examples of lateral movements (Fig. $6 c$; Movie $4 ; n=3$ cochleae; 27 tracks analyzed). Importantly, instantaneous retractions were still common, such that the mean vector continued to point toward the modiolus (Fig. 6c). However, the directionality index was significantly decreased from E16 (Fig. 6e). Because the overall speed increased (Fig. $6 g$ ), these observations suggest that individual branches in the IHC region have begun to make rapid but intermittent retractions in a variety of directions. Consistent with this idea, the trajectories of the branches that retracted (Fig. $7 b$, green), advanced (Fig. $7 b$, yellow), or persisted (Fig. $7 b$, magenta) all appeared more broadly arrayed than at E16 (Fig. 7a).

The behavior of SGN branches in the $\mathrm{OHC}$ region was even more dramatically changed from E16 to E18. Whereas most movements were directed back toward the modiolus at E16 (Fig. $6 b)$, the mean vector for branches growing in the $\mathrm{OHC}$ region shifted away from the ganglion at E18 (Fig. 6d), and this was accompanied by a significant increase in speed $(0.190 \pm 0.014$ $\mu \mathrm{m} / \mathrm{min}$ at E16 vs $0.305 \pm 0.032 \mu \mathrm{m} / \mathrm{min}$ at E18; Fig. $6 g)$. In parallel, the percentage of tracks with consecutive retractions was significantly decreased ( $21 \pm 4.8 \%$; Fig. $6 f)$, consistent with the qualitative change in the nature of their overall trajectories (Fig. $7 d$, green). Instead, many branches advanced within the $\mathrm{OHC}$ region, often covering large distances in short periods of time (Fig. $7 d$, yellow), with others circling locally (Fig. $7 d$, magenta), as would be expected for type II SGNs searching for OHC targets.

One interpretation of these changes in behavior is that the events at E16 contribute to the initial positioning of the SGN processes, whereas those at E18 reflect local exploration and growth by the branches of each type of SGN process within its appropriate target area. If this is true, then the branches already confined to the IHC region at E18 should almost never enter the $\mathrm{OHC}$ region. Indeed, the vast majority of processes (89.9 \pm $6.3 \%$ ) retracted after entering the $\mathrm{OHC}$ region at E18 (Fig. $6 f$ ). This is significantly more than at E16. Thus, most peripheral processes in the OHC region at E18 likely belong to type II SGNs that are advancing among the three rows of OHCs and spiraling toward the base, whereas future type I SGNs are instead extending exploratory branches around the IHCs that leave the $\mathrm{OHC}$ region quickly if they venture in. Although individual branches likely interact with inappropriate targets, they do not seem to persist long enough to form stable contacts.

\section{Processes in the $\mathrm{OHC}$ region at $\mathrm{E} 18$ preferentially retain branches in the type II position}

There are two axes of segregation in the mature cochlea: the radial axis, ie, from IHC to OHC, and the sm-st axis, ie, from the roof to the floor of the cochlea, with type I SGNs consistently closer to sm than the type II SGNs. At E18, SGN processes elaborate many exploratory branches that are frequently retracted from the $\mathrm{OHC}$ region, especially if the branches enter from the IHC region, offering one potential mechanism for segregation along the radial dimension. How are SGN processes further segregated along the sm-st axis? To address this question, we focused on one radial position, ie, the $\mathrm{OHC}$ region, where most of the branches appear to extend from type II SGN processes but others belong to type I SGNs that have not yet removed extra branches. Indeed, at E18, individual branches are positioned at a range of depths in the organ of Corti (Fig. 8a). We predicted that branches in the "type II" position would be retained, whereas those in the "type I" position would be retracted, even if they extend from a type II SGN process. To test this idea, we created 3D reconstructions of individual peripheral processes from the E18 movies, coded for depth along the sm-st axis (Fig. 8b), and followed their behavior over time in the $\mathrm{OHC}$ region (Fig. $8 c$; Movie 5). As predicted, the branches closer to st showed greater persistence. In contrast, those on the sm side exhibited highly dynamic and transient extension followed by retraction (Fig. $8 d$ ). This was true even for branches from a single process, suggesting that there is a preferred substrate for type II SGN process extension on the st side of the organ of Corti. Together, our results suggest that type I and type II SGNs are separated from each other due to differential responses to the OHC environment. Initially, type I SGN pro- 

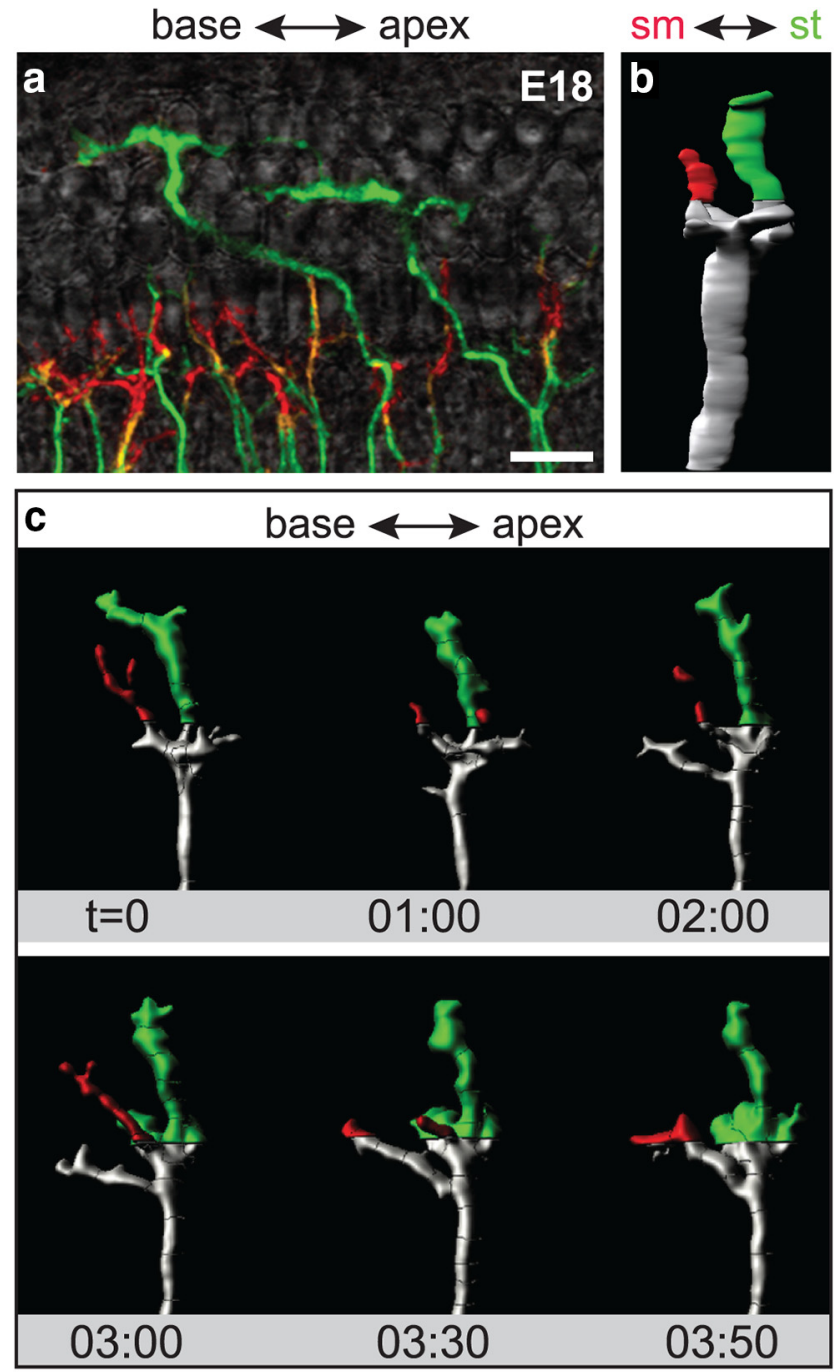

d

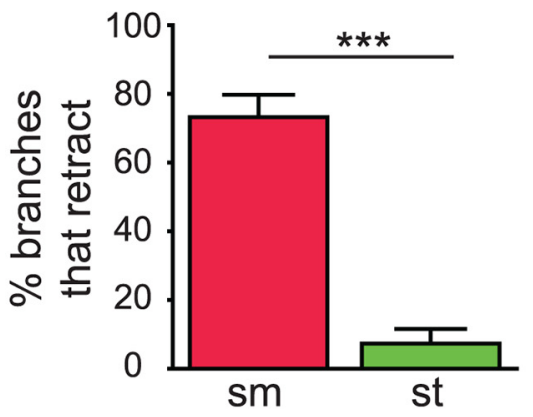

Figure 8. SGN peripheral processes at different depths of the organ of Corti exhibit distinct behaviors in the $\mathrm{OHCregion}$. $\boldsymbol{a}$, Aflat-mountview of az-stack coded for depthillustrates theorganization of $S \mathrm{GN}$ processes in an E18Neurog1-CreER ${ }^{T 2} ; A 114$ cochlea. SGN processes in the sm halfare shown in red, whereas thosein the sthalf are green. $\boldsymbol{b}$, A rotated view of the $3 \mathrm{D}$ reconstruction of an individual process, with sm to the left and st to the right.c, Amontage of framesfrom a movie of the samereconstructed process (Movie5), nowshown in flat-mount. Branches in the $\mathrm{OHC}$ region were coded by depth. The branch closer to sm extended and retracted, whereas the branch positioned closer to st persisted. $\boldsymbol{d}$, Within the $\mathrm{OHC}$ region, $\mathrm{SGN}$ processes in the sm half of the organ of Corti were significantly more likely to retract within $40 \mathrm{~min}$ than those in the st half. (Each region: 4 cochleae; sm region: 38 tracks; st region: 20 tracks; ${ }^{* * *} p<0.0001, \chi^{2}$ test). Scale bar, $20 \mu \mathrm{m}$. Error bars are SEM.

cesses are positioned to the IHC region because the OHC environment is inhospitable, possibly due to the action of repellants. Subsequently, type I processes elaborate branches that explore locally, retracting any off-target branches such that only contacts

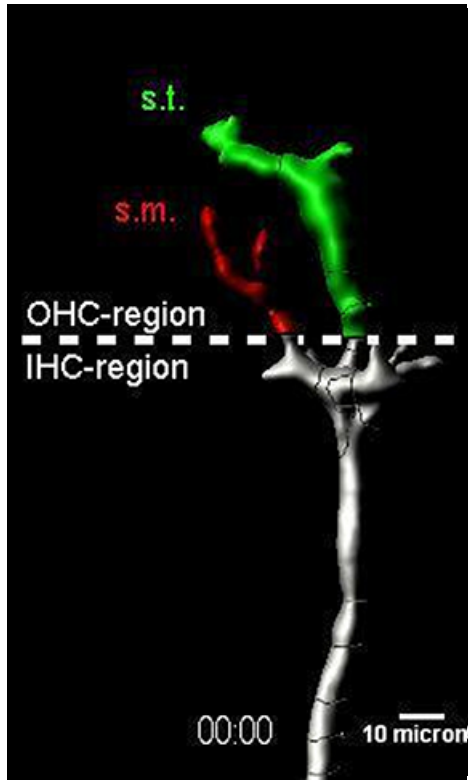

Movie 5. A depth-coded reconstruction of an SGN fiber growing in the $\mathrm{OHC}$ region at E18. A $3 \mathrm{D}$ reconstruction of representative branches from a process in the $\mathrm{OHC}-$-region of an $\mathrm{E} 18 \mathrm{Neu}$ rog1-CreER $R^{T 2}$; Al14 cochlea that was imaged every $10 \mathrm{~min}$. SGN processes are color-coded by their proximity to $\mathrm{sm}$ and st along the $z$-axis. Fibers closest to $\mathrm{sm}(\mathrm{red})$ are highly retractive and transient. Fibers within the st half of the volume persist and expand. Scale bar, $10 \mu \mathrm{m}$.

with IHCs are retained. Type II SGNs, in contrast, are able to remain in the $\mathrm{OHC}$ region and stabilize branches that are closer to the floor of the cochlea, followed by rapid extension toward the base.

\section{Discussion}

Efforts to understand how specificity is achieved in the nervous system have uncovered evidence for at least 10 mechanisms (Sanes and Yamagata, 2009). Yet each circuit holds its own constraints, such as the distance between targets and the degree of precision needed for normal function. We propose that repulsive signals influence the initial separation of SGN processes at E16, such that by E18, neither population makes long lasting contacts with inappropriate targets and only SGN-HC contacts are retained within each region (Fig. 9). Subsequently, other mechanisms likely sculpt the final pattern of connectivity. Our findings argue against a major role for synaptic elimination during targeting and offer an alternative explanation for why type I processes branch outside of the IHC region postnatally despite having been segregated from type II processes during embryogenesis.

Despite the relative simplicity of the cochlea, it has been challenging to pinpoint how different types of SGNs find their targets. Initially, it was proposed that type I SGNs make synapses with $\mathrm{OHCs}$ that are subsequently eliminated (Perkins and Morest, 1975; Echteler, 1992; Simmons, 1994; Huang et al., 2012). However, SGNs with type I and II morphologies can already be recognized shortly after reaching the organ of Corti, days before the onset of overshooting (Bruce et al., 1997; Koundakjian et al., 2007), suggesting that each subtype is guided directly to its target. Using Neurog1-CreER ${ }^{T 2}$ to label SGNs sparsely and hence generate high resolution $3 \mathrm{D}$ reconstructions, we were able to distinguish radial type I-like processes routinely positioned closer to sm from spiraling type II-like processes closer to st (Fig. 2). The segregation by depth held even for branches interacting with the 


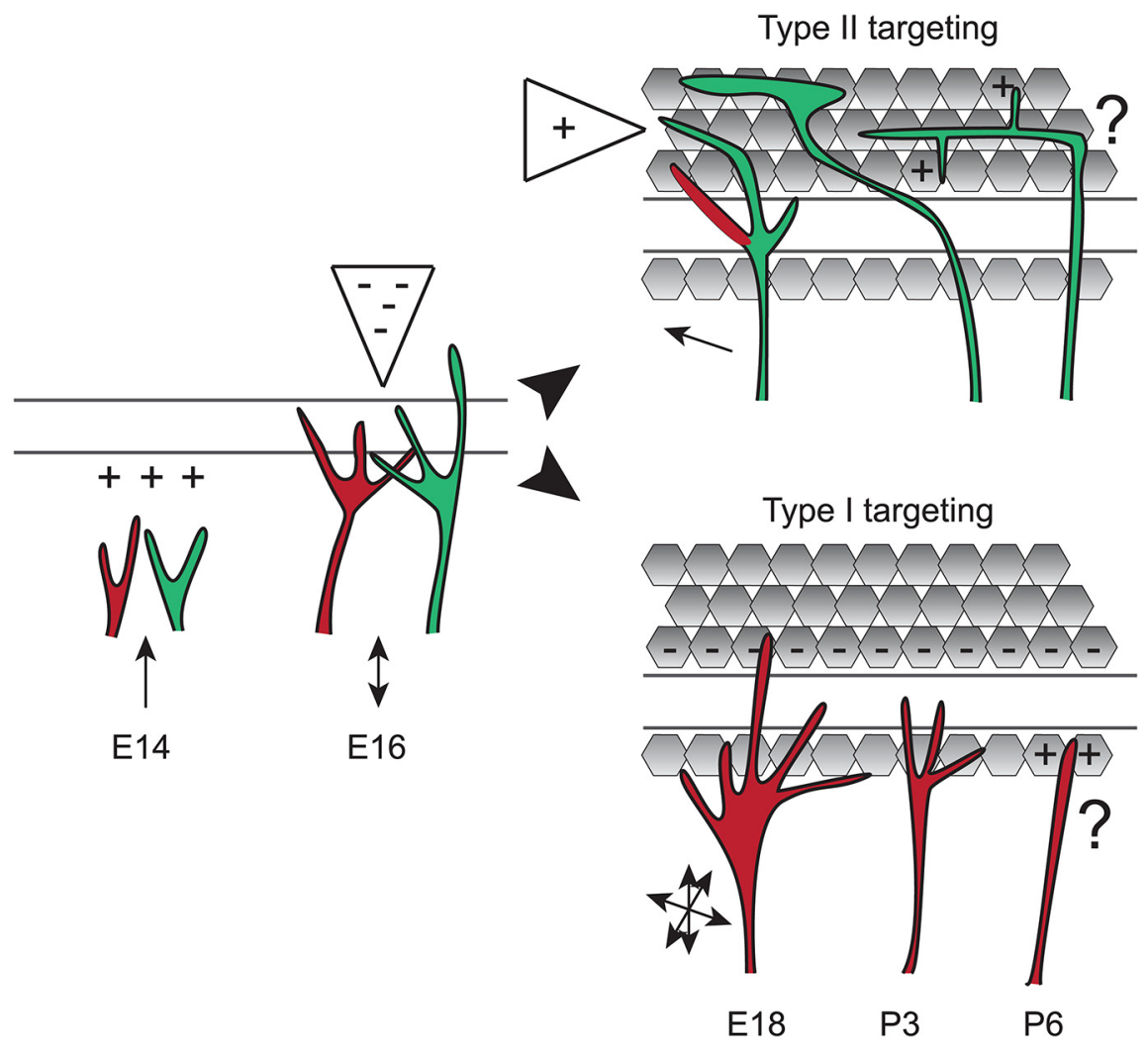

Figure 9. A model for cochlear target selection. We propose that type I (red) and type II (green) SGN fibers are attracted to the cochlea and then positioned in the organ of Corti by diffusible repellants in the OHC region. Subsequently, type II fibers stay in the $\mathrm{OHC}$ region, growing toward attractants and extending branches locally that are retained by positive cues, whereas branches at inappropriate depths (red) are retracted. Type I fibers elaborate many exploratory branches that interact with a variety of cell types, but quickly leave the $\mathrm{OHC}$ region and ultimately retain a single branch contacting an inner hair cell. As a result, type I and type II SGN processes are anatomically segregated in the mature cochlea, both along the radial axis and along the sm-st axis. Additional mechanisms likely direct the stabilization and refinement of synaptic contacts later in development.

same potential $\mathrm{OHC}$ target: branches from radial fibers were reliably located above the spiral fibers (Fig. 3). Although we cannot rule out some misclassification, particularly of type II SGNs that have not yet turned toward the base, the preferential retention of branches closer to st in the OHC region (Fig. 8) suggests that depth is an excellent predictor of identity. Indeed, such anatomical criteria may be more definitive than cell-type-specific markers, which are often transiently coexpressed in immature neurons (Hafidi et al., 1993; Azim et al., 2009).

Our analysis confirmed that the excess branches that arise postnatally belong to radial processes and are gradually removed in favor of those that end near IHCs. Previously, such observations favored a role for synaptic elimination, because the withdrawal of processes from the $\mathrm{OHC}$ region coincided with a loss of synaptic puncta (Huang et al., 2012). However, we found that surprisingly few branches contacted OHCs (Fig. 3), and those that did were quickly (Fig. 6) retracted. On the other hand, most branches already in the OHC region advanced quickly, often toward the base of the cochlea. Strikingly, these distinct behaviors correlated with anatomical position, with branches in the "type I" location retracting and those in the "type II" position persisting (Fig. 8). These findings favor a model where type I and type II SGNs find their targets by responding differentially to the environment, rather than by activity-dependent synaptic elimination. It will be important to test this model independently using alternative methods of SGN classification as they become available.
There are many ways to refine the final wiring pattern of a circuit in addition to synaptic elimination (Schuldiner and Yaron, 2015). Because neither the location of the branches nor the time frame of retraction is consistent with the presence of transient synapses between type I SGNs and $\mathrm{OHCs}$, elimination of type I fibers may not be responsible for the change in presynaptic and postsynaptic protein distribution during the first postnatal week. In fact, all hair cells produce an excess of presynaptic puncta that are localized upon neurite contact (Sobkowicz et al., 1982; Safieddine et al., 2012). Perhaps many of the ribbons are lost just because a postsynaptic process was never present. In addition, ribbons may be juxtaposed to type II SGN processes, which also respond to glutamate (Weisz et al., 2009). In this case, synapse loss could be explained by the massive death of type II SGNs that occurs over this time period (Barclay et al., 2011). Finally, it is important to note that synaptic elimination may influence other aspects of wiring, such as the formation of a single contact between an SGN and an IHC.

Our results provide a cellular framework for understanding the stepwise emergence of connectivity in the cochlea. First, after growing steadily through the mesenchyme (Appler et al., 2013), likely on top of other fibers in the radial bundle (Coate et al., 2012), SGN peripheral processes begin to make forward and reverse movements, as if a balance of attractants and repellants position fibers in the cochlea, regardless of their final destination. This fits with the observation that SGN processes line up together in the organ of Corti at E15.5 and do not persist in the OHC region until E16.5 (Koundakjian et al., 2007). Although it is possible that the $\mathrm{OHC}$ region is nonpermissive because of the absence of an attractive cue, we find that retractive movements are directed and consecutive, even on the modiolar side of the PC border, as if in response to diffusible repulsive cues. Indeed, in flies, growth cones that are repelled from the midline by the secreted protein Slit do not collapse but instead extend branches across the midline that are quickly retracted, often before reaching the midline (Murray and Whitington, 1999). In the cochlea, an analogous activity might be provided by the secreted repellant Semaphorin $3 \mathrm{~F}$, which is produced outside of the IHC region. Intriguingly, the Neuropilin-2 receptor is present on all SGNs, not just on type I neurons (Coate et al., 2015). It seems likely that repellants keep type I processes out of the $\mathrm{OHC}$ region, given the obvious differences in behavior seen in the IHC and $\mathrm{OHC}$ regions by E18; however, it remains unclear whether type II SGNs ignore the repulsive cues from the outset or instead acquire resistance over time.

A different mode of retraction occurs as the two populations seek their appropriate partners. Unlike the steady approach and retreat behaviors at E16, processes in the IHC region extended more exploratory branches at E18. Importantly, those branches that started in the $\mathrm{IHC}$ region and crossed into the $\mathrm{OHC}$ region 
were nearly all immediately withdrawn. In contrast, branches already in the $\mathrm{OHC}$ region advanced quickly or made only occasional retractions that resulted in little net movement. These differential behaviors could reflect the activity of membrane bound repellants, such as EphrinA5, which is expressed only in OHCs (Defourny et al., 2013). Because the relevant receptor, EphA4, is restricted to type I SGNs, only these neurons would be predicted to retract upon contact with OHCs. Thus, diffusible and cell-surface repellants may cooperate to guide type I and type II SGN processes toward their targets and eliminate any off-target branches.

The highly branched morphology of SGN processes, particularly at E18, is reminiscent of other neurons imaged in situ, where target exploration involved frequent extension and retraction of multiple branches (Kaethner and Stuermer, 1992; Godement et al., 1994). Similar effects on branch dynamics are also proposed to influence the topographic positioning of axons in the cortex (Ruthazer et al., 2010) and tectum (Simpson et al., 2013). Indeed, subtypes of retinal ganglion cells show biased growth into the tectum followed by local refinement (Robles et al., 2013). Thus, directed guidance of branches may be common during target selection.

In other systems, attractive molecules clearly play an important instructive role, not only providing a code, but also mediating cell-cell interactions that bias growth toward appropriate targets (Sanes and Yamagata, 2009; Hadjieconomou et al., 2011). Although retractive behaviors dominated in the cochlea, we also observed long-term retention of branches, indicating that attractive cues are involved. This was particularly obvious for the type II-like processes, which grew rapidly toward the base at E18 and showed a strong preference for regions closer to scala tympani. The relevant signals are unknown, but the disorganization of type II SGN innervation in Proxl mutant mice may provide some hints (Fritzsch et al., 2010).

How attractive cues influence type I fibers remains unclear. It could be that repulsion from every other cell type in the local area simply forces fibers to elaborate synapses only with the IHCs. Indeed, when type I fibers persist abnormally within the OHC region, as occurs in Ephrin $A 5$ and EphA4 mutant mice, they seem to form synapses with OHCs (Defourny et al., 2013). This suggests that type I fibers are not selectively attracted to IHCs, but instead that the surrounding cells are unsuitable partners. A similar lack of selectivity has been observed during the reinnervation of hair cells in zebrafish (Pujol-Martí et al., 2014). Thus, repulsion may provide the major source of specificity at the onset of target selection, with additional mechanisms responsible for other important features such as synapse number.

This study highlights the diverse array of strategies that ensure the formation of precisely wired networks. In the retina, $\sim 40$ amacrine cell types establish contacts with $\sim 20$ ganglion cells (Sanes and Zipursky, 2010), a pattern that depends on both retraction and homophilic adhesion mediated by cell-type-specific receptors (D'Orazi et al., 2014). Cochlear wiring offers an example at the opposite extreme: there are only two major subclasses of SGNs and their targets are in distinct locations. In this system, it may suffice to prevent type I SGNs from remaining in the OHC region, with no need for cell-type-specific cues that distinguish IHCs from OHCs. Instead, generic adhesive systems may connect SGNs to their targets. Subsequently, additional mechanisms such as axon-axon competition and activity-dependent refinement may further sculpt connectivity.

\section{References}

Appler JM, Lu CC, Druckenbrod NR, Yu WM, Koundakjian EJ, Goodrich LV (2013) Gata3 is a critical regulator of cochlear wiring. J Neurosci 33: 3679-3691. CrossRef Medline

Azim E, Shnider SJ, Cederquist GY, Sohur US, Macklis JD (2009) Lmo4 and Clim 1 progressively delineate cortical projection neuron subtypes during development. Cereb Cortex 19:i62-i69. CrossRef Medline

Barclay M, Ryan AF, Housley GD (2011) Type I vs type II spiral ganglion neurons exhibit differential survival and neuritogenesis during cochlear development. Neural Dev 6:33. CrossRef Medline

Berglund AM, Ryugo DK (1987) Hair cell innervation by spiral ganglion neurons in the mouse. J Comp Neurol 255:560-570. CrossRef Medline

Bruce LL, Kingsley J, Nichols DH, Fritzsch B (1997) The development of vestibulocochlear efferents and cochlear afferents in mice. Int J Dev Neurosci 15:671-692. CrossRef Medline

Coate TM, Raft S, Zhao X, Ryan AK, Crenshaw EB 3rd, Kelley MW (2012) Otic mesenchyme cells regulate spiral ganglion axon fasciculation through a Pou3f4/EphA4 signaling pathway. Neuron 73:49-63. CrossRef Medline

Coate TM, Spita NA, Zhang KD, Isgrig KT, Kelley MW (2015) Neuropilin2/semaphorin-3F-mediated repulsion promotes inner hair cell innervation by spiral ganglion neurons. eLife 4:e07830. CrossRef

Defourny J, Poirrier AL, Lallemend F, Mateo Sánchez S, Neef J, Vanderhaeghen P, Soriano E, Peuckert C, Kullander K, Fritzsch B, Nguyen L, Moonen G, Moser T, Malgrange B (2013) Ephrin-A5/EphA4 signalling controls specific afferent targeting to cochlear hair cells. Nat Commun 4:1438. CrossRef Medline

D’Orazi FD, Suzuki SC, Wong RO (2014) Neuronal remodeling in retinal circuit assembly, disassembly, and reassembly. Trends Neurosci 37: 594-603. CrossRef Medline

Echteler SM (1992) Developmental segregation in the afferent projections to mammalian auditory hair cells. Proc Natl Acad Sci US A 89: 6324-6327. CrossRef Medline

Fritzsch B, Dillard M, Lavado A, Harvey NL, Jahan I (2010) Canal cristae growth and fiber extension to the outer hair cells of the mouse ear require Proxl activity. PLoS One 5:e9377. CrossRef Medline

Godement P, Wang LC, Mason CA (1994) Retinal axon divergence in the optic chiasm: dynamics of growth cone behavior at the midline. J Neurosci 14:7024-7039. Medline

Gorelik R, Gautreau A (2014) Quantitative and unbiased analysis of directional persistence in cell migration. Nat Protoc 9:1931-1943. CrossRef Medline

Hadjieconomou D, Timofeev K, Salecker I (2011) A step-by-step guide to visual circuit assembly in Drosophila. Curr Opin Neurobiol 21:76-84. CrossRef Medline

Hafidi A, Després G, Romand R (1993) Ontogenesis of type II spiral ganglion neurons during development: peripherin immunohistochemistry. Int J Dev Neurosci 11:507-512. CrossRef Medline

Hong W, Luo L (2014) Genetic control of wiring specificity in the fly olfactory system. Genetics 196:17-29. CrossRef Medline

Huang LC, Thorne PR, Housley GD, Montgomery JM (2007) Spatiotemporal definition of neurite outgrowth, refinement and retraction in the developing mouse cochlea. Development 134:2925-2933. CrossRef Medline

Huang LC, Barclay M, Lee K, Peter S, Housley GD, Thorne PR, Montgomery JM (2012) Synaptic profiles during neurite extension, refinement and retraction in the developing cochlea. Neural Dev 7:38. CrossRef Medline

Kaethner RJ, Stuermer CA (1992) Dynamics of terminal arbor formation and target approach of retinotectal axons in living zebrafish embryos: a time-lapse study of single axons. J Neurosci 12:3257-3271. Medline

Koundakjian EJ, Appler JL, Goodrich LV (2007) Auditory neurons make stereotyped wiring decisions before maturation of their targets. J Neurosci 27:14078-14088. CrossRef Medline

Li GN, Hoffman-Kim D (2008) Evaluation of neurite outgrowth anisotropy using a novel application of circular analysis. J Neurosci Methods 174: 202-214. CrossRef Medline

Lu CC, Cao XJ, Wright S, Ma L, Oertel D, Goodrich LV (2014) Mutation of Npr2 leads to blurred tonotopic organization of central auditory circuits in mice. PLoS Genetics 10:e1004823. CrossRef Medline

Madisen L, Zwingman TA, Sunkin SM, Oh SW, Zariwala HA, Gu H, Ng LL, Palmiter RD, Hawrylycz MJ, Jones AR, Lein ES, Zeng H (2010) A robust and high-throughput Cre reporting and characterization system for the whole mouse brain. Nat Neurosci 13:133-140. CrossRef Medline 
Meyer AC, Moser T (2010) Structure and function of cochlear afferent innervation. Curr Opin Otolaryngol Head Neck Surg 18:441-446. CrossRef Medline

Murray MJ, Whitington PM (1999) Effects of roundabout on growth cone dynamics, filopodial length, and growth cone morphology at the midline and throughout the neuropile. J Neurosci 19:7901-7912. Medline

Pan N, Kopecky B, Jahan I, Fritzsch B (2012) Understanding the evolution and development of neurosensory transcription factors of the ear to enhance therapeutic translation. Cell Tissue Res 349:415-432. CrossRef Medline

Pecot MY, Tadros W, Nern A, Bader M, Chen Y, Zipursky SL (2013) Multiple interactions control synaptic layer specificity in the Drosophila visual system. Neuron 77:299-310. CrossRef Medline

Perkins RE, Morest DK (1975) A study of cochlear innervation patterns in cats and rats with the Golgi method and Nomarkski Optics. J Comp Neurol 163:129-158. CrossRef Medline

Pujol-Martí J, Faucherre A, Aziz-Bose R, Asgharsharghi A, Colombelli J, Trapani JG, López-Schier H (2014) Converging axons collectively initiate and maintain synaptic selectivity in a constantly remodeling sensory organ. Curr Biol 24:2968-2974. CrossRef Medline

Robles E, Filosa A, Baier H (2013) Precise lamination of retinal axons generates multiple parallel input pathways in the tectum. J Neurosci 33: 5027-5039. CrossRef Medline

Ross SE, Mardinly AR, McCord AE, Zurawski J, Cohen S, Jung C, Hu L, Mok SI, Shah A, Savner EM, Tolias C, Corfas R, Chen S, Inquimbert P, Xu Y, McInnes RR, Rice FL, Corfas G, Ma Q, Woolf CJ, Greenberg ME (2010) Loss of inhibitory interneurons in the dorsal spinal cord and elevated itch in Bhlhb5 mutant mice. Neuron 65:886-898. CrossRef Medline

Ruthazer ES, Bachleda AR, Olavarria JF (2010) Role of interstitial branching in the development of visual corticocortical connections: a time-lapse and fixed-tissue analysis. J Comp Neurol 518:4963-4979. CrossRef Medline

Safieddine S, El-Amraoui A, Petit C (2012) The auditory hair cell ribbon synapse: from assembly to function. Annu Rev Neurosci 35:509-528. CrossRef Medline

Sanes JR, Yamagata M (2009) Many paths to synaptic specificity. Annu Rev Cell Dev Biol 25:161-195. CrossRef Medline

Sanes JR, Zipursky SL (2010) Design principles of insect and vertebrate visual systems. Neuron 66:15-36. CrossRef Medline

Schuldiner O, Yaron A (2015) Mechanisms of developmental neurite pruning. Cell Mol Life Sci 72:101-119. CrossRef Medline

Simmons DD (1994) A transient afferent innervation of outer hair cells in the postnatal cochlea. Neuroreport 5:1309-1312. CrossRef Medline

Simpson HD, Kita EM, Scott EK, Goodhill GJ (2013) A quantitative analysis of branching, growth cone turning, and directed growth in zebrafish retinotectal axon guidance. J Comp Neurol 521:1409-1429. CrossRef Medline

Sobkowicz HM, Rose JE, Scott GE, Slapnick SM (1982) Ribbon synapses in the developing intact and cultured organ of Corti in the mouse. J Neurosci 2:942-957. Medline

Sobkowicz HM, Rose JE, Scott GL, Levenick CV (1986) Distribution of synaptic ribbons in the developing organ of Corti. J Neurocytol 15:693-714. CrossRef Medline

Takeuchi H, Sakano H (2014) Neural map formation in the mouse olfactory system. Cell Mol Life Sci 71:3049-3057. CrossRef Medline

Weisz C, Glowatzki E, Fuchs P (2009) The postsynaptic function of type II cochlear afferents. Nature 461:1126-1129. CrossRef Medline

Yu WM, Appler JM, Kim YH, Nishitani AM, Holt JR, Goodrich LV (2013) A Gata3-Mafb transcriptional network directs postsynaptic differentiation in synapses specialized for hearing. eLife 2:e01341. CrossRef Medline 